INSTITUT NATIONAL DE RECHERCHE EN INFORMATIQUE ET EN AUTOMATIQUE

\title{
The Vlasov-Poisson system with strong external magnetic field. Finite Larmor radius regime
}

Mihai Bostan

$\mathbf{N}^{\circ}$ ????

Mars 2007

Thème NUM

apport

de recherche 



\title{
The Vlasov-Poisson system with strong external magnetic field. Finite Larmor radius regime
}

\author{
Mihai Bostan田 \\ Thème NUM — Systèmes numériques \\ Projet Calvi \\ Rapport de recherche $n^{\circ}$ ???? - Mars 2007 - 35 pages
}

\begin{abstract}
We study here the finite Larmor radius regime for the Vlasov-Poisson equations with strong external magnetic field. The derivation of the limit model follows by formal expansion in power series with respect to a small parameter. If we replace the particle distribution by the center distribution of the Larmor circles the limit of these densities satisfies a transport equation, whose velocity is given by the gyro-average of the electric field. We justify rigorously the convergence towards the above model and we investigate the well-posedness of it.
\end{abstract}

Key-words: Vlasov-Maxwell equations, Finite Larmor radius, Gyro-average

\footnotetext{
* Laboratoire de Mathématiques de Besançon, UMR CNRS 6623, Université de Franche-Comté, 16 route de Gray, 25030 Besançon Cedex France et INRIA Lorraine, projet CALVI. E-mail : mbostan@univ-fcomte.fr, mbostan@iecn.u-nancy.fr
} 


\section{Equations de Vlasov-Poisson avec champ magnétique extérieur fort. Régime du rayon de Larmor fini}

Résumé : Nous abordons ici le régime asymptotique du rayon de Larmor fini pour les équations de Vlasov-Poisson avec champ magnétique extérieur fort. Un des points clé consiste à remplacer la densité de particules par la densité de centres de Larmor. La limite de ces densités vérifie une équation de transport dont la vitesse est donnée par la gyro-moyenne du champ électrique.

Mots-clés : Equations de Vlasov-Maxwell, Rayon de Larmor fini, Gyro-moyenne 


\section{Introduction}

The dynamics of a population of charged particles interacting only through electro-magnetic fields created collectively is described by the Vlasov-Maxwell equations. The Cauchy problem for this model is now well understood [13, 17, 18, 19. Nevertheless the existence of global smooth solution for the three dimensional Vlasov-Maxwell system is still a classical open problem. Conditional results for the global existence of strong solutions, depending on the behavior of the support of the particle densities, have been obtained by different approaches in [20, 11], 26]. It is also worth mentioning the recent results when considering data close to equilibrium [23, 31] or reduced models [12, [5].

The numerical resolution of the Vlasov-Maxwell equations is also a challenge problem since we are working in a phase space with three spatial dimensions and three momentum dimensions. Moreover new difficulties appear when studying asymptotic regimes due to the multi-scale character of the problem. Motivated by the magnetic confinement fusion (MCF) the study of strong magnetic field effect is now of crucial importance. Results for the Vlasov or Vlasov-Poisson equations with large external magnetic fields have been investigated recently [15], 21], 16], 8]. The guiding-center approximation for the VlasovMaxwell system with strong initial magnetic field was studied in [7] by the modulated energy method, see also [6, 9], 22] for other applications of this technique.

The asymptotic regime we wish to address here is the gyro-kinetic model with finite Larmor radius. We consider a population of non relativistic electrons whose density is denoted by $f$. We work in the two dimensional setting assuming that $f=f(t, x, p),(t, x, p) \in \mathbb{R}_{+} \times$ $\mathbb{R}^{2} \times \mathbb{R}^{2}$ and that the electro-magnetic field has the form $(E, B)=\left(E_{1}, E_{2}, 0,0,0, B_{3}\right)(t, x)$, $(t, x) \in \mathbb{R}_{+} \times \mathbb{R}^{2}$. The particle density satisfies the Vlasov equation

$$
\partial_{t} f+\frac{p}{m_{e}} \cdot \nabla_{x} f-e\left(E(t, x)+B_{3}(t, x) \frac{\perp p}{m_{e}}\right) \cdot \nabla_{p} f=0,
$$

where $-e<0$ is the electron charge, $m_{e}>0$ is the electron mass and the notation ${ }^{\perp} v$ stands for $\left(v_{2},-v_{1}\right)$ for any $v=\left(v_{1}, v_{2}\right) \in \mathbb{R}^{2}$. The self-consistent electro-magnetic field $(E, B)$ verifies the Maxwell equations

$$
\begin{gathered}
\partial_{t} E-c_{0}^{2 \perp} \nabla_{x} B_{3}=\frac{e}{\varepsilon_{0}} \int_{\mathbb{R}^{2}} \frac{p}{m_{e}} f(t, x, p) d p, \\
\partial_{t} B_{3}+\operatorname{div}_{x}{ }^{\perp} E=0, \\
\operatorname{div}_{x} E=\frac{e}{\varepsilon_{0}}\left(n-\int_{\mathbb{R}^{2}} f(t, x, p) d p\right) .
\end{gathered}
$$

Here $\varepsilon_{0}$ is the vacuum permittivity, $c_{0}$ is the light speed in the vacuum and $n$ is the concentration of the background ion distribution. We introduce the following characteristic scales

$$
t=T t^{\prime}, \quad x=\lambda_{D} x^{\prime}, \quad p=P p^{\prime},
$$

RR $n^{\circ} 0123456789$ 
where $\lambda_{D}=\left(\frac{\varepsilon_{0} K_{B} T_{\mathrm{th}}}{e^{2} n}\right)^{1 / 2}$ is the Debye length, $K_{B}$ is the Boltzmann constant and $T_{\mathrm{th}}$ is the temperature. Consider also the thermal momentum $p_{\text {th }}$ and the inverse of the plasma frequency $T_{p}$ given respectively by

$$
\frac{p_{\mathrm{th}}^{2}}{m_{e}}=K_{B} T_{\mathrm{th}}, \quad T_{p}^{2}=\frac{m_{e} \varepsilon_{0}}{e^{2} n} .
$$

Let us denote by $f^{\prime}, E^{\prime}, B^{\prime}$ the dimensionless unknowns given by

$$
f=\frac{n}{P^{2}} f^{\prime}\left(\frac{t}{T}, \frac{x}{\lambda_{D}}, \frac{p}{P}\right), E=\frac{\lambda_{D} e n}{\varepsilon_{0}} E^{\prime}\left(\frac{t}{T}, \frac{x}{\lambda_{D}}\right), B=\frac{\lambda_{D} e n m_{e}}{\varepsilon^{2} \varepsilon_{0} P} B^{\prime}\left(\frac{t}{T}, \frac{x}{\lambda_{D}}\right) .
$$

Observing that

$$
\frac{\lambda_{D} e n m_{e}}{\varepsilon_{0} P}=\frac{p_{\mathrm{th}} m_{e}}{P e T_{p}}
$$

we also have

$$
B(t, x)=\frac{1}{\varepsilon^{2}} \frac{m_{e} p_{\mathrm{th}}}{e T_{p} P} .
$$

In this case the equations become having dropped the primes

$$
\begin{gathered}
\partial_{t} f+\frac{T}{T_{p}} \frac{P}{p_{\mathrm{th}}} p \cdot \nabla_{x} f-\frac{T}{T_{p}} \frac{p_{\mathrm{th}}}{P}\left(E(t, x)+\frac{{ }^{\perp}}{\varepsilon^{2}} B_{3}(t, x)\right) \cdot \nabla_{p} f=0, \\
\partial_{t} E-\frac{1}{\varepsilon} \frac{T}{T_{p}} \frac{p_{\mathrm{th}}}{P}\left(\frac{m_{e} c_{0}}{p_{\mathrm{th}}}\right)^{2}{ }^{\perp} \nabla_{x}\left(\frac{B_{3}}{\varepsilon}\right)=\frac{T}{T_{p}} \frac{P}{p_{\mathrm{th}}} j(t, x), \\
\partial_{t}\left(\frac{B_{3}}{\varepsilon}\right)+\varepsilon \frac{T}{T_{p}} \frac{P}{p_{\mathrm{th}}} \operatorname{div}_{x}^{\perp} E=0, \\
\operatorname{div}_{x} E=1-\rho(t, x),
\end{gathered}
$$

where $\rho=\int_{\mathbb{R}^{2}} f d p, j=\int_{\mathbb{R}^{2}} p f d p$. We choose the reference units such that

$$
\frac{T}{T_{p}} \frac{P}{p_{\mathrm{th}}}=\frac{1}{\varepsilon^{2}}, \frac{T}{T_{p}} \frac{p_{\mathrm{th}}}{P}=1,
$$

which means $\frac{T}{T_{p}}=\frac{P}{p_{\mathrm{th}}}=\frac{1}{\varepsilon}$. Note that in this case the unit for the magnetic field becomes $\frac{1}{\varepsilon} \frac{m_{e}}{e T_{p}}$ and the Larmor radius corresponding to this magnetic field and the reference velocity $\frac{P}{m_{e}}$ is given by $R=T_{p} \frac{p_{\mathrm{th}}}{m_{e}}=\lambda_{D}$. Up to a multiplication constant $\left(\frac{m_{e} c_{0}}{p_{\mathrm{th}}}\right)^{2}$ we obtain the following system, known as the finite Larmor radius regime see [14]

$$
\partial_{t} f^{\varepsilon}+\frac{p}{\varepsilon^{2}} \cdot \nabla_{x} f^{\varepsilon}-\left(E^{\varepsilon}(t, x)+B_{3}^{\varepsilon}(t, x) \frac{{ }^{\perp} p}{\varepsilon^{2}}\right) \cdot \nabla_{p} f^{\varepsilon}=0,
$$




$$
\begin{gathered}
\partial_{t} E^{\varepsilon}-\frac{1}{\varepsilon}{ }^{\perp} \nabla_{x}\left(\frac{B_{3}^{\varepsilon}}{\varepsilon}\right)=\frac{j^{\varepsilon}(t, x)}{\varepsilon^{2}}, \\
\partial_{t}\left(\frac{B_{3}^{\varepsilon}}{\varepsilon}\right)+\frac{1}{\varepsilon} \operatorname{div}_{x}^{\perp} E^{\varepsilon}=0, \\
\operatorname{div}_{x} E^{\varepsilon}=1-\rho^{\varepsilon}(t, x) .
\end{gathered}
$$

We address here the case of a large external constant magnetic field $B_{3}^{\varepsilon}=B_{0,3} \neq 0$ i.e., we investigate the Vlasov-Poisson equations

$$
\begin{gathered}
\partial_{t} f^{\varepsilon}+\frac{p}{\varepsilon^{2}} \cdot \nabla_{x} f^{\varepsilon}-\left(E^{\varepsilon}(t, x)+B_{0,3} \frac{{ }^{\perp} p}{\varepsilon^{2}}\right) \cdot \nabla_{p} f^{\varepsilon}=0, \\
\operatorname{div}_{x} E^{\varepsilon}=1-\rho^{\varepsilon}(t, x), \quad \operatorname{div}_{x}{ }^{\perp} E^{\varepsilon}=0,
\end{gathered}
$$

with the initial condition

$$
f^{\varepsilon}(0, x, p)=f_{0}^{\varepsilon}(x, p) .
$$

In order to simplify our analysis we assume periodicity with respect to the space variable $x \in \mathbb{T}^{2}$, where $\mathbb{T}^{2}=\mathbb{R}^{2} / \mathbb{Z}^{2}$ is equipped with the restriction of the Lebesgue measure of $\mathbb{R}^{2}$ on $\left[0,1\left[{ }^{2}\right.\right.$. We are searching for electric fields $E^{\varepsilon}$ deriving from space periodic potentials $\Phi^{\varepsilon}$ satisfying

$$
E^{\varepsilon}=\nabla_{x} \Phi^{\varepsilon}, \quad \Delta_{x} \Phi^{\varepsilon}=1-\rho^{\varepsilon}(t, x), \quad(t, x) \in \mathbb{R}_{+} \times \mathbb{T}^{2} .
$$

The above problem has solution provided that the global neutrality condition is satisfied i.e., $\int_{\mathbb{T}^{2}} \rho^{\varepsilon}(t, x) d x=1$. Note also that the solution is unique up to a constant. We consider here zero average solutions $\int_{\mathbb{T}^{2}} \Phi^{\varepsilon}(t, x) d x=0$. There are in the literature a lot of studies concerning the Cauchy problem for the Vlasov-Poisson system. The existence of weak solutions has been studied in [1, 25]. For smooth solutions the reader can refer to [30, 24], [2], 29], 27], 28].

One of the key points when analyzing (9) is to replace the particle distribution functions $f^{\varepsilon}$ by the center distribution functions $g^{\varepsilon}$ given by

$$
g^{\varepsilon}(t, y, p)=f^{\varepsilon}(t, x, p),
$$

where $y=x-\frac{\perp p}{B_{0,3}}$ is the center of the Larmor circle. Performing the above change of unknown yields the equation

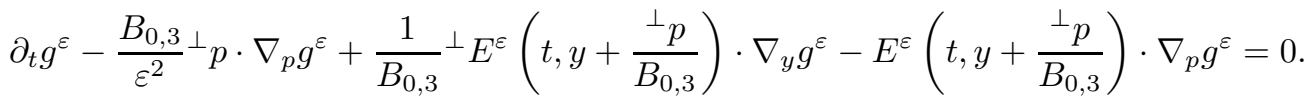

Observing that

$$
\frac{1}{B_{0,3}} \operatorname{div}_{y}\left\{{ }^{\perp} E^{\varepsilon}\left(t, y+\frac{{ }^{\perp} p}{B_{0,3}}\right)\right\}=\operatorname{div}_{p}\left\{E^{\varepsilon}\left(t, y+\frac{{ }^{\perp} p}{B_{0,3}}\right)\right\},
$$

$\mathrm{RR} \mathrm{n}^{\circ} 0123456789$ 
the above equation can be written in the conservative form

$$
\partial_{t} g^{\varepsilon}-\frac{B_{0,3}}{\varepsilon^{2}} \operatorname{div}_{p}\left(g^{\varepsilon \perp} p\right)+\operatorname{div}_{y}\left(g^{\varepsilon} \frac{\perp E^{\varepsilon}(t, x)}{B_{0,3}}\right)-\operatorname{div}_{p}\left(g^{\varepsilon} E^{\varepsilon}(t, x)\right)=0,
$$

supplemented with the initial condition

$$
g^{\varepsilon}(0, y, p)=f_{0}^{\varepsilon}\left(y+\frac{{ }^{\perp} p}{B_{0,3}}, p\right), \quad(y, p) \in \mathbb{T}^{2} \times \mathbb{R}^{2} .
$$

As $\varepsilon$ goes to zero, we expect that the limit of the center distributions $\left(g^{\varepsilon}\right)_{\varepsilon>0}$ becomes radial symmetric with respect to $p \in \mathbb{R}^{2}$. Indeed, passing formally to the limit as $\varepsilon \searrow 0$ in (12) we deduce that ${ }^{\perp} p \cdot \nabla_{p} g=0$, where $g=\lim _{\varepsilon \backslash 0} g^{\varepsilon}$. Eventually, the transport equation satisfied by $g$ comes by writing the weak formulation of (12) with test functions $\psi=\psi(t, y, p)$

having radial symmetry with respect to $p \in \mathbb{R}^{2}$, since in this case the singular term vanishes

$$
-\left\langle\frac{B_{0,3}}{\varepsilon^{2}} \operatorname{div}_{p}\left(g^{\varepsilon \perp} p\right), \psi\right\rangle_{\mathcal{D}^{\prime}, \mathcal{D}}=\int_{\mathbb{R}_{+}} \int_{\mathbb{T}^{2}} \int_{\mathbb{R}^{2}} \frac{B_{0,3}}{\varepsilon^{2}} g^{\varepsilon \perp} p \cdot \nabla_{p} \psi d p d y d t=0 .
$$

The evaluations of the other terms

$$
\left\langle\operatorname{div}_{y}\left(g^{\varepsilon} \frac{\perp E^{\varepsilon}(t, x)}{B_{0,3}}\right), \psi\right\rangle_{\mathcal{D}^{\prime}, \mathcal{D}},\left\langle\operatorname{div}_{p}\left(g^{\varepsilon} E^{\varepsilon}(t, x)\right), \psi\right\rangle_{\mathcal{D}^{\prime}, \mathcal{D}}
$$

lead naturally to the gyro-average operator which will play an important role in the analysis of the finite radius Larmor regime.

The paper is organized as follows. In Section 2 we establish uniform estimates with respect to the small parameter $\varepsilon>0$. These estimates come basically from the conservation of the total energy, combined with Sobolev and interpolation inequalities. Section 3 is devoted to the formal derivation of the limit model. We employ here the Hilbert method, by expanding the solution in power series of some small parameter. The well-posedness of the limit model is studied in the next section. We establish existence and uniqueness results for the strong solution. The convergence towards the limit model is proved rigorously in Section 5. We obtain strong convergence in $L^{2}$ for well prepared initial conditions. In the last section we investigate higher order approximations. We identify formally the equations satisfied by the first order correction terms.

\section{Uniform estimates}

We work with smooth solutions $\left(f^{\varepsilon}, E^{\varepsilon}\right)_{\varepsilon>0}$ for the Vlasov-Poisson problem (9), (10), (11). For further computations we establish a priori estimates with respect to the parameter $\varepsilon>0$. Besides the smoothness of the initial conditions $\left(f_{0}^{\varepsilon}\right)_{\varepsilon>0}$ we make the following assumptions 
H1) $f_{0}^{\varepsilon} \geq 0, \quad \int_{\mathbb{T}^{2}} \int_{\mathbb{R}^{2}} f_{0}^{\varepsilon}(x, p) d p d x=1, \forall \varepsilon>0$;

H2) $\sup _{\varepsilon>0} \frac{1}{2} \int_{\mathbb{T}^{2}} \int_{\mathbb{R}^{2}}|p|^{2} f_{0}^{\varepsilon}(x, p) d p d x<+\infty$;

H3) there is a bounded non increasing function $F_{0} \in L^{\infty}\left(\mathbb{R}_{+}\right) \cap L^{1}\left(\mathbb{R}_{+} ; r d r\right)$ such that $f_{0}^{\varepsilon}(x, p) \leq F_{0}(|p|), \forall(x, p) \in \mathbb{T}^{2} \times \mathbb{R}^{2}, \varepsilon>0$.

Integrating with respect to the momentum the Vlasov equation yields the continuity equation

$$
\partial_{t} \rho^{\varepsilon}+\frac{1}{\varepsilon^{2}} \operatorname{div}_{x} j^{\varepsilon}=0
$$

where $\rho^{\varepsilon}=\int_{\mathbb{R}^{2}} f^{\varepsilon} d p, j^{\varepsilon}=\int_{\mathbb{R}^{2}} p f^{\varepsilon} d p$. We deduce easily by H1 that the global neutrality condition holds true for any $t \in \mathbb{R}_{+}$

$$
\int_{\mathbb{T}^{2}} \int_{\mathbb{R}^{2}} f^{\varepsilon}(t, x, p) d p d x=1, \forall \varepsilon>0 .
$$

By standard arguments we deduce that the total energy is conserved on $\mathbb{R}_{+}$

$$
\frac{d}{d t}\left\{\frac{1}{2} \int_{\mathbb{T}^{2}} \int_{\mathbb{R}^{2}}|p|^{2} f^{\varepsilon}(t, x, p) d p d x+\frac{\varepsilon^{2}}{2} \int_{\mathbb{T}^{2}}\left|E^{\varepsilon}(t, x)\right|^{2} d x\right\}=0 .
$$

Usual computations involving interpolation inequalities provide an estimate for the $L^{2}$ norm of the charge densities $\left(\rho^{\varepsilon}\right)_{\varepsilon>0}$.

$$
\left\|\rho^{\varepsilon}(t)\right\|_{L^{2}\left(\mathbb{T}^{2}\right)} \leq\left(8 \pi\left\|f_{0}^{\varepsilon}\right\|_{L^{\infty}}\right)^{1 / 2}\left(\int_{\mathbb{T}^{2}} \int_{\mathbb{R}^{2}} \frac{|p|^{2}}{2} f^{\varepsilon}(t, x, p) d p d x\right)^{1 / 2}, \quad t \in \mathbb{R}_{+}, \quad \varepsilon>0 .
$$

In particular $\mathrm{H} 2, \mathrm{H} 3$ imply that

$$
\sup _{\varepsilon>0}\left\|\rho_{0}^{\varepsilon}\right\|_{L^{2}(\mathbb{T})}<+\infty, \sup _{\varepsilon>0}\left\|E_{0}^{\varepsilon}\right\|_{H^{1}\left(\mathbb{T}^{2}\right)}<+\infty
$$

and by (14) we deduce that

$$
\sup _{0<\varepsilon<1, t \in \mathbb{R}_{+}}\left\{\frac{1}{2} \int_{\mathbb{T}^{2}} \int_{\mathbb{R}^{2}}|p|^{2} f^{\varepsilon}(t, x, p) d p d x+\int_{\mathbb{T}^{2}}\left|\rho^{\varepsilon}(t, x)\right|^{2} d x\right\}<+\infty,
$$

and

$$
\sup _{0<\varepsilon<1, t \in \mathbb{R}_{+}}\left\|E^{\varepsilon}(t)\right\|_{H^{1}\left(\mathbb{T}^{2}\right)}<+\infty .
$$

Actually following the ideas in [4] it is possible to obtain more uniform bounds with respect to the parameter $\varepsilon$. For any $R>0$ we can write

$$
\rho^{\varepsilon}(t, x)=\rho_{1}^{\varepsilon}(t, x)+\rho_{2}^{\varepsilon}(t, x),
$$

$\mathrm{RR} \quad \mathrm{n}^{\circ} 0123456789$ 
with $\rho_{1}^{\varepsilon}:=\int_{\mathbb{R}^{2}} f^{\varepsilon} \mathbf{1}_{\{|p| \leq 2 R\}} d p, \rho_{2}^{\varepsilon}:=\int_{\mathbb{R}^{2}} f^{\varepsilon} \mathbf{1}_{\{|p|>2 R\}} d p$. In order to estimate the charge density $\rho_{1}^{\varepsilon}$ we combine the Hölder inequality and the bound for the kinetic energy. For any $\eta>0$ we denote by $(2+\eta)^{\prime}$ the conjugate exponent of $2+\eta$ i.e., $\frac{1}{2+\eta}+\frac{1}{(2+\eta)^{\prime}}=1$. We have

$$
\rho_{1}^{\varepsilon}(t, x) \leq\left(\int_{|p| \leq 2 R}|p|^{2} f^{\varepsilon} d p\right)^{\frac{1}{2+\eta}}\left(\int_{|p| \leq 2 R}|p|^{-2 \frac{(2+\eta)^{\prime}}{2+\eta}} f^{\varepsilon} d p\right)^{\frac{1}{(2+\eta)^{\prime}}} .
$$

Taking into account that $\left\|f^{\mathcal{\varepsilon}}\right\|_{L^{\infty}} \leq\left\|F_{0}\right\|_{L^{\infty}}$ we have

$$
\int_{|p| \leq 2 R}|p|^{-2 \frac{(2+\eta)^{\prime}}{2+\eta}} f^{\varepsilon} d p \leq\left\|F_{0}\right\|_{L^{\infty}} 2 \pi \int_{0}^{2 R} r^{-2 \frac{(2+\eta)^{\prime}}{2+\eta}} r d r
$$

and therefore, by using the bound for the kinetic energy, we deduce that

$$
\left\|\rho_{1}^{\varepsilon}\right\|_{L^{2+\eta}\left(\mathbb{T}^{2}\right)} \leq C_{1} R^{\frac{2 \eta}{2 \eta \eta}}, \quad t \in \mathbb{R}_{+}, \quad 0<\varepsilon<1,
$$

for some constant $C_{1}$ not depending on $R$ or $t$. For estimating the charge density $\rho_{2}^{\varepsilon}$ observe that

$$
f^{\varepsilon}(t, x, p)=f_{0}^{\varepsilon}\left(X^{\varepsilon}(0 ; t, x, p), P^{\varepsilon}(0 ; t, x, p)\right),
$$

where $\left(X^{\varepsilon}, P^{\varepsilon}\right)$ solve the characteristic system

$$
\frac{d X^{\varepsilon}}{d s}=\frac{P^{\varepsilon}(s)}{\varepsilon^{2}}, \frac{d P^{\varepsilon}}{d s}=-\left(E^{\varepsilon}\left(s, X^{\varepsilon}(s)\right)+B_{0,3} \frac{\perp P^{\varepsilon}(s)}{\varepsilon^{2}}\right),
$$

and the conditions $\left(X^{\varepsilon}, P^{\varepsilon}\right)(t ; t, x, p)=(x, p)$. Multiplying the second characteristic equation by $P^{\varepsilon}(s)$ we obtain easily that $\left|\frac{d}{d s}\right| P^{\varepsilon}(s)|| \leq\left\|E^{\varepsilon}(s)\right\|_{L^{\infty}\left(\mathbb{T}^{2}\right)^{2}}$ implying that

$$
\left|P^{\varepsilon}(0 ; t, x, p)\right| \geq|p|-t\left\|E^{\varepsilon}\right\|_{L^{\infty}(] 0, t\left[\times \mathbb{T}^{2}\right)} .
$$

We fix now $t>0$ and let us consider $R=R(t)=t\left\|E^{\varepsilon}\right\|_{L^{\infty}(] 0, t\left[\times \mathbb{T}^{2}\right)}$. The hypothesis H3 yields for any $s \in[0, t]$

$$
\begin{aligned}
\rho_{2}^{\varepsilon}(s, x) & =\int_{|p|>2 R} f_{0}^{\varepsilon}\left(X^{\varepsilon}(0 ; s, x, p), P^{\varepsilon}(0 ; s, x, p)\right) d p \\
& \leq \int_{|p|>2 R} F_{0}(|p|-R) d p \\
& \leq 4 \pi \int_{R}^{+\infty} F_{0}(r) r d r \\
& \leq 4 \pi \int_{0}^{+\infty} r F_{0}(r) d r=: C_{2} .
\end{aligned}
$$

Finally combining (18), (20) and Sobolev inequalities we deduce

$$
\left\|E^{\varepsilon}(s)\right\|_{L^{\infty}\left(\mathbb{T}^{2}\right)} \leq C_{3}\left\|\rho^{\varepsilon}(s)\right\|_{L^{2+\eta}\left(\mathbb{T}^{2}\right)} \leq C(t, \eta)\left(1+\left\|E^{\varepsilon}\right\|_{L^{\infty}(] 0, t\left[\times \mathbb{T}^{2}\right)}^{\frac{2 \eta}{2+\eta}}\right), \forall s \in[0, t],
$$


and by taking $0<\eta<2$ we obtain that the following estimate holds

$$
\sup _{0<\varepsilon<1}\left\|E^{\varepsilon}\right\|_{L^{\infty}(] 0, t\left[\times \mathbb{T}^{2}\right)}<+\infty, \quad t \in \mathbb{R}_{+} .
$$

In particular, for any $s \in[0, t], \varepsilon \in] 0,1[$ we have

$$
\left\|\rho_{1}^{\varepsilon}(s)\right\|_{L^{\infty}\left(\mathbb{T}^{2}\right)} \leq 4 \pi(R(s))^{2}\left\|f^{\varepsilon}\right\|_{L^{\infty}} \leq 4 \pi t^{2}\left\|E^{\varepsilon}\right\|_{L^{\infty}(] 0, t\left[\times \mathbb{T}^{2}\right)}^{2}\left\|F_{0}\right\|_{L^{\infty}},
$$

and thus, thanks to (17), (20) we deduce that

$$
\sup _{0<\varepsilon<1}\left\|\rho^{\varepsilon}\right\|_{L^{\infty}(] 0, t\left[\times \mathbb{T}^{2}\right)}<+\infty, \quad t \in \mathbb{R}_{+} .
$$

Since $f^{\varepsilon}$ belongs to $L^{\infty}\left(\mathbb{R}_{+} ; L^{1}\left(\mathbb{T}^{2} \times \mathbb{R}^{2}\right)\right) \cap L^{\infty}\left(\mathbb{R}_{+} ; L^{\infty}\left(\mathbb{T}^{2} \times \mathbb{R}^{2}\right)\right)$ we have also $f^{\varepsilon} \in$ $L^{\infty}\left(\mathbb{R}_{+} ; L^{2}\left(\mathbb{T}^{2} \times \mathbb{R}^{2}\right)\right)$. Multiplying the Vlasov equation (9) by $f^{\varepsilon}$ it is easily seen that

$$
\int_{\mathbb{T}^{2}} \int_{\mathbb{R}^{2}}\left(f^{\varepsilon}\right)^{2}(t, x, p) d p d x=\int_{\mathbb{T}^{2}} \int_{\mathbb{R}^{2}}\left(f_{0}^{\varepsilon}\right)^{2}(x, p) d p d x, \quad t \in \mathbb{R}_{+}, \varepsilon>0 .
$$

The above conclusions are summarized up in the following proposition

Proposition 2.1 Assume that $\left(f_{0}^{\varepsilon}\right)_{\varepsilon>0}$ are smooth initial conditions satisfying the hypotheses H1, H2, H3. Let $\left(f^{\varepsilon}, E^{\varepsilon}\right)_{\varepsilon>0}$ be the smooth solutions for the Vlasov-Poisson system (9), 10), 11). Then we have

$$
\begin{gathered}
\int_{\mathbb{T}^{2}} \int_{\mathbb{R}^{2}} f^{\varepsilon}(t) d p d x=1, \int_{\mathbb{T}^{2}} \int_{\mathbb{R}^{2}}\left(f^{\varepsilon}\right)^{2}(t) d p d x=\int_{\mathbb{T}^{2}} \int_{\mathbb{R}^{2}}\left(f_{0}^{\varepsilon}\right)^{2} d p d x, \quad t \in \mathbb{R}_{+}, \quad \varepsilon>0, \\
\sup _{0<\varepsilon<1, t \in \mathbb{R}_{+}}\left\{\frac{1}{2} \int_{\mathbb{T}^{2}} \int_{\mathbb{R}^{2}}|p|^{2} f^{\varepsilon}(t, x, p) d p d x+\left\|E^{\varepsilon}(t)\right\|_{H^{1}\left(\mathbb{T}^{2}\right)}^{2}\right\}<+\infty, \\
\sup _{0<\varepsilon<1}\left\|\rho^{\varepsilon}\right\|_{L^{\infty}(] 0, T\left[\times \mathbb{T}^{2}\right)}<+\infty, \sup _{0<\varepsilon<1}\left\|E^{\varepsilon}\right\|_{L^{\infty}(] 0, T\left[; W^{1, q}\left(\mathbb{T}^{2}\right)\right)}<+\infty, T \in \mathbb{R}_{+}, 1 \leq q<\infty .
\end{gathered}
$$

Remark 2.1 It is easily seen by using (19) that if $\operatorname{supp} f_{0}^{\varepsilon} \subset \mathbb{T}^{2} \times B_{R}$, then $f^{\varepsilon}(t)$ remains compactly supported, uniformly for $t$ in compact sets of $\mathbb{R}_{+}$and

$$
\operatorname{supp} f^{\varepsilon}(t) \subset \mathbb{T}^{2} \times B_{R(T)}, \quad t \in[0, T],
$$

with $R(T)=R+T\left\|E^{\varepsilon}\right\|_{L^{\infty}(] 0, T\left[\times \mathbb{T}^{2}\right)}$.

\section{Formal derivation of the limit model}

We consider the asymptotic expansions in powers of $\varepsilon^{2}$ (notice that $\varepsilon^{2}$ is the small parameter appearing in (9))

$$
f^{\varepsilon}=f+\varepsilon^{2} f_{1}+\varepsilon^{4} f_{2}+\ldots,
$$

$\mathrm{RR} \quad \mathrm{n}^{\circ} 0123456789$ 


$$
E^{\varepsilon}=E+\varepsilon^{2} E_{1}+\varepsilon^{4} E_{2}+\ldots .
$$

Plugging these ansatz in the Vlasov equation (9) yields

$$
\begin{gathered}
p \cdot \nabla_{x} f-B_{0,3}{ }^{\perp} p \cdot \nabla_{p} f=0, \\
\partial_{t} f-E(t, x) \cdot \nabla_{p} f+p \cdot \nabla_{x} f_{1}-B_{0,3}{ }^{\perp} p \cdot \nabla_{p} f_{1}=0, \\
\partial_{t} f_{1}-E(t, x) \cdot \nabla_{p} f_{1}-E_{1}(t, x) \cdot \nabla_{p} f+p \cdot \nabla_{x} f_{2}-B_{0,3}{ }^{\perp} p \cdot \nabla_{p} f_{2}=0,
\end{gathered}
$$

From the Poisson equation we deduce also that

$$
\operatorname{div}_{x} E=1-\rho, \operatorname{div}_{x} E_{1}=-\rho_{1}, \quad \ldots
$$

where $\rho=\int_{\mathbb{R}^{2}} f d p$ and $\rho_{k}=\int_{\mathbb{R}^{2}} f_{k} d p$ for any $k \geq 1$. If we denote by $j,\left(j_{k}\right)_{k \geq 1}$ the current densities $j=\int_{\mathbb{R}^{2}} p f d p, j_{k}=\int_{\mathbb{R}^{2}} p f_{k} d p$ for any $k \geq 1$ it is easily seen that the following continuity equations hold

$$
\operatorname{div}_{x} j=0, \quad \partial_{t} \rho+\operatorname{div}_{x} j_{1}=0, \partial_{t} \rho_{1}+\operatorname{div}_{x} j_{2}=0, \ldots
$$

And finally notice that

$$
\operatorname{div}_{x}{ }^{\perp} E=0, \operatorname{div}_{x}{ }^{\perp} E_{1}=0, \ldots
$$

Introducing the differential operator $\mathcal{T}=p \cdot \nabla_{x}-B_{0,3}{ }^{\perp} p \cdot \nabla_{p}$ the previous equations can be written

$$
\begin{gathered}
\mathcal{T} f=0, \\
\partial_{t} f-E(t, x) \cdot \nabla_{p} f+\mathcal{T} f_{1}=0, \\
\partial_{t} f_{1}-E(t, x) \cdot \nabla_{p} f_{1}-E_{1}(t, x) \cdot \nabla_{p} f+\mathcal{T} f_{2}=0,
\end{gathered}
$$

Performing the change of coordinates $(x, p) \rightarrow\left(y=x-\frac{\perp^{\perp}}{B_{0,3}}, p\right)$ simplifies the expression of the operator $\mathcal{T}$. Indeed, for any $u=u(x, p)$ let us denote by $v=v(y, p)$ the function

$$
v(y, p)=u(x, p), \quad x=y+\frac{{ }^{\perp} p}{B_{0,3}} .
$$

Applying the chain rule yields

$$
\nabla_{x} u(x, p)=\nabla_{y} v(y, p)
$$




$$
\nabla_{p} u(x, p)=\nabla_{p} v(y, p)+{\frac{1}{B_{0,3}}}^{\perp} \nabla_{y} v(y, p)
$$

In particular we have

$$
\mathcal{T} u(x, p)=-B_{0,3}{ }^{\perp} p \cdot \nabla_{p} v(y, p)
$$

The above change of coordinates facilitates the study of the operator $\mathcal{T}$.

Proposition 3.1 Let $u \in L_{\text {loc }}^{1}\left(\mathbb{T}^{2} \times \mathbb{R}^{2}\right)$. Then $\mathcal{T} u=0$ in $\mathcal{D}^{\prime}\left(\mathbb{T}^{2} \times \mathbb{R}^{2}\right)$ iff there is a function $w \in L_{\text {loc }}^{1}\left(\mathbb{T}^{2} \times \mathbb{R}_{+} ; r d y d r\right)$ such that $u(x, p)=w\left(x-\frac{\perp p}{B_{0,3}},|p|\right)$ for a.a. $(x, p) \in \mathbb{T}^{2} \times \mathbb{R}^{2}$.

Proof. For any function $\varphi \in C_{c}^{1}\left(\mathbb{T}^{2} \times \mathbb{R}^{2}\right)$ we have

$$
\int_{\mathbb{T}^{2}} \int_{\mathbb{R}^{2}} u(x, p) \mathcal{T} \varphi(x, p) d p d x=0 .
$$

Let us denote by $v$ and $\psi$ the functions

$$
v(y, p)=u\left(y+\frac{{ }^{\perp} p}{B_{0,3}}, p\right), \psi(y, p)=\varphi\left(y+\frac{{ }^{\perp} p}{B_{0,3}}, p\right), \quad(y, p) \in \mathbb{T}^{2} \times \mathbb{R}^{2} .
$$

After changing the variables $(x, p)$ by $(y, p)$ the equality (35) implies

$$
\int_{\mathbb{T}^{2}} \int_{\mathbb{R}^{2}} v(y, p){ }^{\perp} p \cdot \nabla_{p} \psi(y, p) d p d y=0 .
$$

Notice that

$$
\frac{\mathrm{d}}{\mathrm{d} \theta} \psi(y, r \cos \theta, r \sin \theta)=-{ }^{\perp} p \cdot \nabla_{p} \psi(y, p), \quad p=(r \cos \theta, r \sin \theta)
$$

and thus we obtain

$$
\int_{\mathbb{T}^{2}} d y \int_{\mathbb{R}_{+}} r d r \int_{0}^{2 \pi} v(y, r \cos \theta, r \sin \theta) \frac{\mathrm{d}}{\mathrm{d} \theta} \psi(y, r \cos \theta, r \sin \theta) d \theta=0,
$$

saying that there is a function $w \in L_{\text {loc }}^{1}\left(\mathbb{T}^{2} \times \mathbb{R}_{+} ; r d y d r\right)$ such that $v(y, p)=w(y,|p|)$ for a.a. $(y, p) \in \mathbb{T}^{2} \times \mathbb{R}^{2}$. Therefore we have

$$
u(x, p)=v\left(x-\frac{{ }^{\perp} p}{B_{0,3}}, p\right)=w\left(x-\frac{{ }^{\perp} p}{B_{0,3}},|p|\right), \quad(x, p) \in \mathbb{T}^{2} \times \mathbb{R}^{2}
$$

$\mathrm{RR} \mathrm{n}^{\circ} 0123456789$ 
Remark 3.1 For any $q \in[1,+\infty]$ we can define $\mathcal{T}$ as linear unbounded operator on $L^{q}\left(\mathbb{T}^{2} \times\right.$ $\mathbb{R}^{2}$ ), with the domain

$$
\mathrm{D}_{q}=\left\{u \in L^{q}\left(\mathbb{T}^{2} \times \mathbb{R}^{2}\right): p \cdot \nabla_{x} u-B_{0,3}{ }^{\perp} p \cdot \nabla_{p} u \in L^{q}\left(\mathbb{T}^{2} \times \mathbb{R}^{2}\right)\right\}
$$

and $\mathcal{T} u=p \cdot \nabla_{x} u-B_{0,3}{ }^{\perp} p \cdot \nabla_{p} u$ for any $u \in \mathrm{D}_{q}$. Obviously, if $u \in \mathrm{D}_{q}$ satisfies $\mathcal{T} u=0$ then $u \in L_{\text {loc }}^{1}\left(\mathbb{T}^{2} \times \mathbb{R}^{2}\right)$ and $\mathcal{T} u=0$ in $\mathcal{D}^{\prime}\left(\mathbb{T}^{2} \times \mathbb{R}^{2}\right)$. Therefore, thanks to Proposition [3.1] the kernel of the operator $\mathcal{T}$ in $L^{q}\left(\mathbb{T}^{2} \times \mathbb{R}^{2}\right)$ setting is given by

$$
\left\{u \in L^{q}\left(\mathbb{T}^{2} \times \mathbb{R}^{2}\right): \exists w \in L^{q}\left(\mathbb{T}^{2} \times \mathbb{R}_{+} ; r d y d r\right), \quad u(x, p)=w\left(x-\frac{{ }^{\perp} p}{B_{0,3}},|p|\right) \text { a.e. }(x, p)\right\}
$$

We introduce now the gyro-average operator \langle\rangle given by

$$
\langle u\rangle(y, p)=\frac{1}{2 \pi} \int_{0}^{2 \pi} u\left(y_{1}+\frac{|p| \sin \theta}{B_{0,3}}, y_{2}-\frac{|p| \cos \theta}{B_{0,3}},|p| \cos \theta,|p| \sin \theta\right) d \theta
$$

for any function $u=u(x, p)$. Actually, with the notation

$$
A(\theta)=\left(\begin{array}{rr}
\cos \theta & -\sin \theta \\
\sin \theta & \cos \theta
\end{array}\right), \theta \in \mathbb{R}
$$

we have for any $\theta_{0} \in \mathbb{R}$

$$
\langle u\rangle(y, p)=\frac{1}{2 \pi} \int_{0}^{2 \pi} u\left(y+\frac{\perp\left(A\left(\theta+\theta_{0}\right) p\right)}{B_{0,3}}, A\left(\theta+\theta_{0}\right) p\right) d \theta .
$$

In particular (taking $\theta_{0}$ such that $A\left(\theta_{0}\right) p=(-|p|, 0)^{t}$ ) we have

$$
\langle u\rangle(y, p)=\frac{1}{2 \pi} \int_{0}^{2 \pi} u\left(y_{1}-\frac{|p| \sin \theta}{B_{0,3}}, y_{2}+\frac{|p| \cos \theta}{B_{0,3}},-|p| \cos \theta,-|p| \sin \theta\right) d \theta .
$$

The gyro-average operator has the following properties

Proposition 3.2 i) The gyro-average operator is linear and continuous from $L^{q}\left(\mathbb{T}^{2} \times \mathbb{R}^{2}\right) \rightarrow$ $L^{q}\left(\mathbb{T}^{2} \times \mathbb{R}^{2}\right)$ and $\|\langle\rangle\|_{\mathcal{L}\left(L^{q}\left(\mathbb{T}^{2} \times \mathbb{R}^{2}\right), L^{q}\left(\mathbb{T}^{2} \times \mathbb{R}^{2}\right)\right)} \leq 1$ for any $q \in[1,+\infty]$.

ii) For any function $u \in L^{q}\left(\mathbb{T}^{2} \times \mathbb{R}^{2}\right), q \in[1,+\infty]$ satisfying $\mathcal{T} u=0$ we have

$$
\langle u\rangle(y, p)=u\left(y+\frac{{ }^{\perp} p}{B_{0,3}}, p\right), \quad(y, p) \in \mathbb{T}^{2} \times \mathbb{R}^{2} .
$$

iii) For any function $u \in W^{1,1}\left(\mathbb{T}^{2} \times \mathbb{R}^{2}\right)$ satisfying $\mathcal{T} u=0$ and $E \in L^{\infty}\left(\mathbb{T}^{2}\right)$ satisfying $\operatorname{div}_{x}{ }^{\perp} E=0$ we have

$$
\left\langle E \cdot \nabla_{p} u\right\rangle(y, p)=-\left\langle\frac{{ }^{\perp} E}{B_{0,3}}\right\rangle(y, p) \cdot \nabla_{x} u\left(y+\frac{{ }^{\perp} p}{B_{0,3}}, p\right) .
$$

iv) If $\left.\partial_{t} u, u \in L^{q}(] 0, T\left[\times \mathbb{T}^{2} \times \mathbb{R}^{2}\right), q \in\right] 1,+\infty[$, then

$$
\partial_{t}\langle u\rangle \in L^{q}(] 0, T\left[\times \mathbb{T}^{2} \times \mathbb{R}^{2}\right), \quad \partial_{t}\langle u\rangle=\left\langle\partial_{t} u\right\rangle .
$$


Proof. i) The case $q=+\infty$ is immediate and we have $\|\langle u\rangle\|_{L^{\infty}} \leq\|u\|_{L^{\infty}}$ for any $u \in$ $L^{\infty}\left(\mathbb{T}^{2} \times \mathbb{R}^{2}\right)$. If $q \in[1,+\infty[$ we have by Hölder inequality

$$
|\langle u\rangle|^{q}(y, p) \leq \frac{1}{2 \pi} \int_{0}^{2 \pi}|u|^{q}\left(y_{1}+\frac{|p| \sin \theta}{B_{0,3}}, y_{2}-\frac{|p| \cos \theta}{B_{0,3}},|p| \cos \theta,|p| \sin \theta\right) d \theta
$$

and after integration over $\mathbb{T}^{2} \times \mathbb{R}^{2}$ one gets

$$
\begin{aligned}
\|\langle u\rangle\|_{L^{q}}^{q} & \leq \frac{1}{2 \pi} \int_{\mathbb{T}^{2}} \int_{\mathbb{R}^{2}} \int_{0}^{2 \pi}|u|^{q}\left(y_{1}+\frac{|p| \sin \theta}{B_{0,3}}, y_{2}-\frac{|p| \cos \theta}{B_{0,3}},|p| \cos \theta,|p| \sin \theta\right) d \theta d p d y \\
& =\frac{1}{2 \pi} \int_{0}^{2 \pi} \int_{\mathbb{T}^{2}} \int_{\mathbb{R}^{2}}|u|^{q}(x,|p| \cos \theta,|p| \sin \theta) d p d x d \theta \\
& =\int_{\mathbb{T}^{2}} \int_{0}^{2 \pi} \int_{\mathbb{R}_{+}}|u|^{q}(x, r \cos \theta, r \sin \theta) r d r d \theta d x \\
& =\|u\|_{L^{q}}^{q} .
\end{aligned}
$$

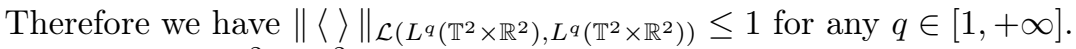

ii) Let $u \in L^{q}\left(\mathbb{T}^{2} \times \mathbb{R}^{2}\right)$ be a function such that $\mathcal{T} u=0$. Therefore by Proposition 3.1 (see also Remark 3.1) we know that $(y, p) \rightarrow u\left(y+\frac{{ }^{\perp} p}{B_{0,3}}, p\right)$ has radial symmetry with respect to $p \in \mathbb{R}^{2}$. We have

$$
\begin{aligned}
\langle u\rangle(y, p) & =\frac{1}{2 \pi} \int_{0}^{2 \pi} u\left(y_{1}+\frac{|p| \sin \theta}{B_{0,3}}, y_{2}-\frac{|p| \cos \theta}{B_{0,3}},|p| \cos \theta,|p| \sin \theta\right) d \theta \\
& =u\left(y+\frac{{ }^{\perp} p}{B_{0,3}}, p\right) .
\end{aligned}
$$

iii) We start by checking the statement for smooth functions $u \in C_{c}^{1}\left(\mathbb{T}^{2} \times \mathbb{R}^{2}\right)$ and $E \in$ $C^{0}\left(\mathbb{T}^{2}\right)$. We have

$$
\left\langle E \cdot \nabla_{p} u\right\rangle(y, p)=\frac{1}{2 \pi} \int_{0}^{2 \pi}\left(E \cdot \nabla_{p} u\right)\left(y_{1}+\frac{|p| \sin \theta}{B_{0,3}}, y_{2}-\frac{|p| \cos \theta}{B_{0,3}},|p| \cos \theta,|p| \sin \theta\right) d \theta .
$$

By formula (33) we obtain

$$
\nabla_{p} u\left(y+\frac{{ }^{\perp} p}{B_{0,3}}, p\right)=\nabla_{p} v(y, p)+{\frac{1}{B_{0,3}}}^{\perp} \nabla_{y} v(y, p), \quad v(y, p)=u(x, p), \quad y=x-\frac{{ }^{\perp} p}{B_{0,3}}
$$

and therefore

$$
\begin{aligned}
\left\langle E \cdot \nabla_{p} u\right\rangle & =\frac{1}{2 \pi} \int_{0}^{2 \pi} E\left(y_{1}+\frac{|p| \sin \theta}{B_{0,3}}, y_{2}-\frac{|p| \cos \theta}{B_{0,3}}\right) \cdot \nabla_{p} v(y,|p| \cos \theta,|p| \sin \theta) d \theta \\
& -\frac{1}{2 \pi B_{0,3}} \int_{0}^{2 \pi} E\left(y_{1}+\frac{|p| \sin \theta}{B_{0,3}}, y_{2}-\frac{|p| \cos \theta}{B_{0,3}}\right) \cdot \nabla_{y} v(y,|p| \cos \theta,|p| \sin \theta) d \theta \\
& =: \quad I_{1}-I_{2} .
\end{aligned}
$$

RR $\quad n^{\circ} 0123456789$ 
Since $\mathcal{T} u=0$ we deduce by Proposition 3.1 that $v$ has radial symmetry with respect to $p \in \mathbb{R}^{2}$, that is, there is $w \in C_{c}^{1}\left(\mathbb{T}^{2} \times \mathbb{R}_{+}\right)$satisfying $\partial_{r} w(\cdot, 0)=0$ such that $v(y, p)=w(y,|p|)$. Therefore

$$
\nabla_{p} v(y, p)=\partial_{r} w(y,|p|) \frac{p}{|p|} .
$$

Let us denote by $\Phi$ the potential of $E$, i.e., $E=\nabla_{x} \Phi, \Phi \in C^{1}\left(\mathbb{T}^{2}\right)$. Notice that we have the equality

$$
\frac{d}{d \theta} \Phi\left(y_{1}+\frac{r \sin \theta}{B_{0,3}}, y_{2}-\frac{r \cos \theta}{B_{0,3}}\right)=\frac{E}{B_{0,3}}\left(y_{1}+\frac{r \sin \theta}{B_{0,3}}, y_{2}-\frac{r \cos \theta}{B_{0,3}}\right) \cdot(r \cos \theta, r \sin \theta) .
$$

Combining these computations we deduce that $I_{1}$ vanishes

$$
\begin{aligned}
I_{1} & =\frac{1}{2 \pi} \int_{0}^{2 \pi} E\left(y_{1}+\frac{|p| \sin \theta}{B_{0,3}}, y_{2}-\frac{|p| \cos \theta}{B_{0,3}}\right) \cdot(\cos \theta, \sin \theta) \partial_{r} w(y,|p|) d \theta \\
& =\frac{B_{0,3}}{|p|} \partial_{r} w(y,|p|) \frac{1}{2 \pi} \int_{0}^{2 \pi} \frac{d}{d \theta} \Phi\left(y_{1}+\frac{|p| \sin \theta}{B_{0,3}}, y_{2}-\frac{|p| \cos \theta}{B_{0,3}}\right) d \theta \\
& =0 .
\end{aligned}
$$

In order to compute the second integral $I_{2}$, observe that $\nabla_{y} v$ has also radial symmetry with respect to $p \in \mathbb{R}^{2}$ and thus

$$
\begin{aligned}
I_{2} & =\nabla_{y} v(y, p) \frac{1}{2 \pi} \int_{0}^{2 \pi \perp} \frac{{ }^{\perp} E}{B_{0,3}}\left(y_{1}+\frac{|p| \sin \theta}{B_{0,3}}, y_{2}-\frac{|p| \cos \theta}{B_{0,3}}\right) d \theta \\
& =\left\langle\frac{{ }^{\perp} E}{B_{0,3}}\right\rangle(y, p) \cdot \nabla_{y} v(y, p) .
\end{aligned}
$$

Finally we obtain

$$
\left\langle E \cdot \nabla_{p} u\right\rangle(y, p)=-\left\langle\frac{{ }^{\perp} E}{B_{0,3}}\right\rangle(y, p) \cdot \nabla_{x} u\left(y+\frac{{ }^{\perp} p}{B_{0,3}}, p\right) .
$$

It remains to prove that the above formula holds true for $u \in W^{1,1}\left(\mathbb{T}^{2} \times \mathbb{R}^{2}\right)$ such that $\mathcal{T} u=0$ and $E \in L^{\infty}\left(\mathbb{T}^{2}\right)$. By regularization arguments we construct the sequences $\left(E_{k}\right)_{k} \subset C^{0}\left(\mathbb{T}^{2}\right)$ such that $\lim _{k \rightarrow+\infty} E_{k}=E$ weakly $\star$ in $L^{\infty}\left(\mathbb{T}^{2}\right)$ and $\left(u_{k}\right)_{k} \subset C_{c}^{1}\left(\mathbb{T}^{2} \times \mathbb{R}^{2}\right)$ such that $\mathcal{T} u_{k}=0$ for any $k, \lim _{k \rightarrow+\infty} u_{k}=u$ strongly in $W^{1,1}\left(\mathbb{T}^{2} \times \mathbb{R}^{2}\right)$. Indeed, since $\mathcal{T} u=0$ there is $w$ such that $u(x, p)=w\left(x-\frac{\perp_{p}}{B_{0,3}},|p|\right)$ satisfying $w, \nabla_{y} w, \partial_{r} w \in L^{1}\left(\mathbb{T}^{2} \times \mathbb{R}_{+} ; r d y d r\right)$. Take now a sequence of smooth functions $\left(w_{k}\right)_{k}$ verifying

$$
\lim _{k \rightarrow+\infty} w_{k}=w, \quad \lim _{k \rightarrow+\infty} \nabla_{y} w_{k}=\nabla_{y} w, \quad \lim _{k \rightarrow+\infty} \partial_{r} w_{k}=\partial_{r} w \text { in } L^{1}\left(\mathbb{T}^{2} \times \mathbb{R}_{+} ; r d y d r\right)
$$

and observe that the sequence $\left(u_{k}(x, p)=w_{k}\left(x-\frac{\perp_{p}}{B_{0,3}},|p|\right)\right)_{k}$ belongs to the kernel of $\mathcal{T}$ and converges towards $u$ strongly in $W^{1,1}\left(\mathbb{T}^{2} \times \mathbb{R}^{2}\right)$. By the previous computations we have 
for any $k$

$$
\left\langle E_{k} \cdot \nabla_{p} u_{k}\right\rangle(y, p)=-\left\langle\frac{{ }^{\perp} E_{k}}{B_{0,3}}\right\rangle(y, p) \cdot \nabla_{x} u_{k}\left(y+\frac{{ }^{\perp} p}{B_{0,3}}, p\right) .
$$

It is easily seen that $\lim _{k \rightarrow+\infty}\left(E_{k} \cdot \nabla_{p} u_{k}\right)=E \cdot \nabla_{p} u$ weakly in $L^{1}\left(\mathbb{T}^{2} \times \mathbb{R}^{2}\right)$. Since the gyroaverage is strongly continuous from $L^{1}\left(\mathbb{T}^{2} \times \mathbb{R}^{2}\right)$ to $L^{1}\left(\mathbb{T}^{2} \times \mathbb{R}^{2}\right)$ it is also weakly continuous from $L^{1}\left(\mathbb{T}^{2} \times \mathbb{R}^{2}\right)$ to $L^{1}\left(\mathbb{T}^{2} \times \mathbb{R}^{2}\right)$ (see 10] Theorem III 9, pp. 39) and therefore

$$
\lim _{k \rightarrow+\infty}\left\langle E_{k} \cdot \nabla_{p} u_{k}\right\rangle=\left\langle E \cdot \nabla_{p} u\right\rangle \text { weakly in } L^{1}\left(\mathbb{T}^{2} \times \mathbb{R}^{2}\right) .
$$

It is easily seen that $\left\langle{ }^{\perp} E_{k}\right\rangle_{k}$ converges weakly $\star$ in $L^{\infty}\left(\mathbb{T}^{2} \times \mathbb{R}^{2}\right)$ towards $\left\langle{ }^{\perp} E\right\rangle$. Indeed, for any function $\varphi \in L^{1}\left(\mathbb{T}^{2} \times \mathbb{R}^{2}\right)$ we have

$$
\begin{aligned}
2 \pi \int_{\mathbb{T}^{2}} \int_{\mathbb{R}^{2}}\left\langle{ }^{\perp} E_{k}\right\rangle & \cdot \varphi d p d y=\int_{\mathbb{T}^{2}} \int_{\mathbb{R}^{2}} \varphi \cdot \int_{0}^{2 \pi} E_{k}\left(y_{1}+\frac{|p| \sin \theta}{B_{0,3}}, y_{2}-\frac{|p| \cos \theta}{B_{0,3}}\right) d \theta d p d y \\
& =\int_{\mathbb{T}^{2}}{ }^{\perp} E_{k}(x) \cdot \int_{\mathbb{R}^{2}} \int_{0}^{2 \pi} \varphi\left(x_{1}-\frac{|p| \sin \theta}{B_{0,3}}, x_{2}+\frac{|p| \cos \theta}{B_{0,3}}, p\right) d \theta d p d x \\
& \rightarrow \int_{\mathbb{T}^{2}}{ }^{\perp} E(x) \cdot \int_{\mathbb{R}^{2}} \int_{0}^{2 \pi} \varphi\left(x_{1}-\frac{|p| \sin \theta}{B_{0,3}}, x_{2}+\frac{|p| \cos \theta}{B_{0,3}}, p\right) d \theta d p d x \\
& =2 \pi \int_{\mathbb{T}^{2}} \int_{\mathbb{R}^{2}}\left\langle{ }^{\perp} E\right\rangle \cdot \varphi d p d y
\end{aligned}
$$

since $\lim _{k \rightarrow+\infty} E_{k}=E$ weakly $\star$ in $L^{\infty}\left(\mathbb{T}^{2}\right)$ and the function

$$
x \rightarrow \int_{\mathbb{R}^{2}} \int_{0}^{2 \pi} \varphi\left(x_{1}-\frac{|p| \sin \theta}{B_{0,3}}, x_{2}+\frac{|p| \cos \theta}{B_{0,3}}, p\right) d \theta d p
$$

belongs to $L^{1}\left(\mathbb{T}^{2}\right)$. Combining now the weak $\star$ convergence in $L^{\infty}\left(\mathbb{T}^{2} \times \mathbb{R}^{2}\right)$ of $\left\langle{ }^{\perp} E_{k}\right\rangle_{k}$ with the strong convergence in $L^{1}\left(\mathbb{T}^{2} \times \mathbb{R}^{2}\right)$ of $\left(\nabla_{x} u_{k}\right)_{k}$ we deduce that

$$
\lim _{k \rightarrow+\infty}\left\langle\frac{{ }^{\perp} E_{k}}{B_{0,3}}\right\rangle(y, p) \cdot \nabla_{x} u_{k}\left(y+\frac{{ }^{\perp} p}{B_{0,3}}, p\right)=\left\langle\frac{{ }^{\perp} E}{B_{0,3}}\right\rangle(y, p) \cdot \nabla_{x} u\left(y+\frac{{ }^{\perp} p}{B_{0,3}}, p\right)
$$

weakly in $L^{1}\left(\mathbb{T}^{2} \times \mathbb{R}^{2}\right)$. Putting together (38), (39), (40) yields our conclusion. iv) Let $\varepsilon>0$ and $h \in \mathbb{R}$ such that $|h|<\varepsilon$. Then

$$
\begin{aligned}
\int_{\varepsilon}^{T-\varepsilon}\|\langle u(t+h)\rangle-\langle u(t)\rangle\|_{L^{q}\left(\mathbb{T}^{2} \times \mathbb{R}^{2}\right)}^{q} d t & \leq \int_{\varepsilon}^{T-\varepsilon}\|u(t+h)-u(t)\|_{L^{q}\left(\mathbb{T}^{2} \times \mathbb{R}^{2}\right)}^{q} d t \\
& \leq|h|^{q}\left\|\partial_{t} u\right\|_{L^{q}(] 0, T\left[\times \mathbb{T}^{2} \times \mathbb{R}^{2}\right)}^{q}
\end{aligned}
$$

saying that $\partial_{t}\langle u\rangle$ belongs to $L^{q}(] 0, T\left[\times \mathbb{T}^{2} \times \mathbb{R}^{2}\right)$ and $\left\|\partial_{t}\langle u\rangle\right\|_{L^{q}} \leq\left\|\partial_{t} u\right\|_{L^{q}}$. Take now a sequence of smooth functions $\left(u_{k}\right)_{k}$ such that $u_{k} \rightarrow u$ and $\partial_{t} u_{k} \rightarrow \partial_{t} u$ in $L^{q}(] 0, T\left[\times \mathbb{T}^{2} \times \mathbb{R}^{2}\right)$. Since $\left(u_{k}\right)_{k}$ are smooth we have $\partial_{t}\left\langle u_{k}\right\rangle=\left\langle\partial_{t} u_{k}\right\rangle$. By the above computation we have

$$
\left\|\partial_{t}\langle u\rangle-\partial_{t}\left\langle u_{k}\right\rangle\right\|_{L^{q}} \leq\left\|\partial_{t} u-\partial_{t} u_{k}\right\|_{L^{q}} \rightarrow 0 \text { as } k \rightarrow+\infty
$$

RR $\quad n^{\circ} 0123456789$ 
and therefore

$$
\partial_{t}\langle u\rangle=\lim _{k \rightarrow+\infty} \partial_{t}\left\langle u_{k}\right\rangle=\lim _{k \rightarrow+\infty}\left\langle\partial_{t} u_{k}\right\rangle=\left\langle\partial_{t} u\right\rangle \text { in } L^{q}(] 0, T\left[\times \mathbb{T}^{2} \times \mathbb{R}^{2}\right) .
$$

We introduce also the operator

$$
[v](x, p)=\frac{1}{2 \pi} \int_{0}^{2 \pi} v\left(x-\frac{{ }^{\perp} p}{B_{0,3}},|p| \cos \alpha,|p| \sin \alpha\right) d \alpha .
$$

Proposition 3.3 i) The operator [] is linear and continuous from $L^{q}\left(\mathbb{T}^{2} \times \mathbb{R}^{2}\right)$ to $L^{q}\left(\mathbb{T}^{2} \times\right.$ $\left.\mathbb{R}^{2}\right)$ and $\|[]\|_{\mathcal{L}\left(L^{q}\left(\mathbb{T}^{2} \times \mathbb{R}^{2}\right), L^{q}\left(\mathbb{T}^{2} \times \mathbb{R}^{2}\right)\right)} \leq 1$ for any $q \in[1,+\infty]$.

ii) For any functions $u \in L^{q}\left(\mathbb{T}^{2} \times \mathbb{R}^{2}\right), v \in L^{q^{\prime}}\left(\mathbb{T}^{2} \times \mathbb{R}^{2}\right), 1 / q+1 / q^{\prime}=1$ we have the equality

$$
\int_{\mathbb{T}^{2}} \int_{\mathbb{R}^{2}}\langle u\rangle(y, p) v(y, p) d p d y=\int_{\mathbb{T}^{2}} \int_{\mathbb{R}^{2}} u(x, p)[v](x, p) d p d x .
$$

iii) For any functions $u \in L^{q}\left(\mathbb{T}^{2} \times \mathbb{R}^{2}\right), v \in L^{q^{\prime}}\left(\mathbb{T}^{2} \times \mathbb{R}^{2}\right), 1 / q+1 / q^{\prime}=1$ with radial symmetry with respect to $p \in \mathbb{R}^{2}$ we have the equality

$$
\int_{\mathbb{T}^{2}} \int_{\mathbb{R}^{2}}\langle u\rangle(y, p) v(y, p) d p d y=\int_{\mathbb{T}^{2}} \int_{\mathbb{R}^{2}} u(x, p)\langle v\rangle(x, p) d p d x .
$$

iv) If $q=2$ the adjoint of the gyro-average operator is [] and the gyro-average operator is symmetric on the subspace of radial symmetric functions with respect to $p \in \mathbb{R}^{2}$.

Proof. i) The assertion is clear for $q=+\infty$. Assume now that $q \in[1,+\infty[$. By Hölder inequality we have

$$
|[v]|^{q}(x, p) \leq \frac{1}{2 \pi} \int_{0}^{2 \pi}|v|^{q}\left(x-\frac{{ }^{\perp} p}{B_{0,3}},|p| \cos \alpha,|p| \sin \alpha\right) d \alpha
$$

and thus after integration over $\mathbb{T}^{2} \times \mathbb{R}^{2}$ one gets

$$
\begin{aligned}
\|[v]\|_{L^{q}}^{q} & \leq \frac{1}{2 \pi} \int_{\mathbb{T}^{2}} \int_{\mathbb{R}^{2}} \int_{0}^{2 \pi}|v|^{q}\left(x-\frac{{ }^{\perp} p}{B_{0,3}},|p| \cos \alpha,|p| \sin \alpha\right) d \alpha d p d x \\
& =\frac{1}{2 \pi} \int_{\mathbb{T}^{2}} \int_{\mathbb{R}^{2}} \int_{0}^{2 \pi}|v|^{q}(y,|p| \cos \alpha,|p| \sin \alpha) d \alpha d p d y \\
& =\|v\|_{L^{q}\left(\mathbb{T}^{2} \times \mathbb{R}^{2}\right)}^{q} .
\end{aligned}
$$

INRIA 
Therefore we have $\|[]\|_{\mathcal{L}\left(L^{q}\left(\mathbb{T}^{2} \times \mathbb{R}^{2}\right), L^{q}\left(\mathbb{T}^{2} \times \mathbb{R}^{2}\right)\right)} \leq 1$ for any $q \in[1,+\infty]$.

ii) For any functions $u \in L^{q}\left(\mathbb{T}^{2} \times \mathbb{R}^{2}\right), v \in L^{q^{\prime}}\left(\mathbb{T}^{2} \times \mathbb{R}^{2}\right), 1 / q+1 / q^{\prime}=1$ we can write

$$
\begin{aligned}
& 2 \pi \int_{\mathbb{T}^{2}} \int_{\mathbb{R}^{2}}\langle u\rangle(y, p) v(y, p) d p d y \\
= & \int_{\mathbb{T}^{2}} \int_{\mathbb{R}^{2}} v(y, p) \int_{0}^{2 \pi} u\left(y_{1}+\frac{|p| \sin \theta}{B_{0,3}}, y_{2}-\frac{|p| \cos \theta}{B_{0,3}},|p| \cos \theta,|p| \sin \theta\right) d \theta d p d y \\
= & \int_{\mathbb{T}^{2}} \int_{\mathbb{R}^{2}} \int_{0}^{2 \pi} u(x,|p| \cos \theta,|p| \sin \theta) v\left(x_{1}-\frac{|p| \sin \theta}{B_{0,3}}, x_{2}+\frac{|p| \cos \theta}{B_{0,3}}, p\right) d \theta d p d y \\
= & \int_{\mathbb{T}^{2}} d x \int_{\mathbb{R}_{+}} r d r \int_{0}^{2 \pi} \int_{0}^{2 \pi} u(x, r \cos \theta, r \sin \theta) v\left(x_{1}-\frac{r \sin \theta}{B_{0,3}}, x_{2}+\frac{r \cos \theta}{B_{0,3}}, r \cos \alpha, r \sin \alpha\right) d \theta d \alpha \\
= & \int_{\mathbb{T}^{2}} \int_{\mathbb{R}^{2}} \int_{0}^{2 \pi} u(x, p) v\left(x-\frac{{ }^{\prime} p}{B_{0,3}},|p| \cos \alpha,|p| \sin \alpha\right) d \alpha d p d x \\
= & 2 \pi \int_{\mathbb{T}^{2}} \int_{\mathbb{R}^{2}} u(x, p)[v](x, p) d p d x .
\end{aligned}
$$

iii) If $v \in L^{q^{\prime}}\left(\mathbb{T}^{2} \times \mathbb{R}^{2}\right)$ has radial symmetry with respect to $p \in \mathbb{R}^{2}$ then

$$
[v](x, p)=v\left(x-\frac{{ }^{\perp} p}{B_{0,3}},-p\right) .
$$

Since $u(x, p)=\tilde{u}(x,|p|)$ we obtain by the previous assertion and (37)

$$
\begin{aligned}
\int_{\mathbb{T}^{2}} \int_{\mathbb{R}^{2}} & \langle u\rangle(y, p) v(y, p) d p d y=\int_{\mathbb{T}^{2}} \int_{\mathbb{R}^{2}} u(x, p)[v](x, p) d p d x \\
= & \int_{\mathbb{T}^{2}} \int_{\mathbb{R}^{2}} u(x, p) v\left(x-\frac{\perp^{2}}{B_{0,3}},-p\right) d p d x \\
= & \int_{\mathbb{T}^{2}} \int_{\mathbb{R}_{+}} \int_{0}^{2 \pi} \tilde{u}(x, r) v\left(x_{1}-\frac{r \sin \theta}{B_{0,3}}, x_{2}+\frac{r \cos \theta}{B_{0,3}},-r \cos \theta,-r \sin \theta\right) r d \theta d r d x \\
= & \int_{\mathbb{T}^{2}} \int_{\mathbb{R}^{2}} \tilde{u}(x,|p|)\langle v\rangle(x, p) d p d x \\
= & \int_{\mathbb{T}^{2}} \int_{\mathbb{R}^{2}} u(x, p)\langle v\rangle(x, p) d p d x .
\end{aligned}
$$

iv) It is a direct consequence of ii) and iii) with $q=2$.

In the following proposition we determine the range of the operator $\mathcal{T}$.

Proposition 3.4 i) Let $R$ belong to $L^{q}\left(\mathbb{T}^{2} \times \mathbb{R}^{2}\right)$ for some $q \in[1,+\infty]$ and assume that there is $u \in L^{q}\left(\mathbb{T}^{2} \times \mathbb{R}^{2}\right)$ such that $\mathcal{T} u=R$. Then $\langle R\rangle=0$.

$\mathrm{RR} \mathrm{n}^{\circ} 0123456789$ 
ii) Let $R$ belong to $L^{q}\left(\mathbb{T}^{2} \times \mathbb{R}^{2}\right)$ for some $q \in[1,+\infty]$, satisfying $\langle R\rangle=0$. Then there is a unique $u \in L^{q}\left(\mathbb{T}^{2} \times \mathbb{R}^{2}\right)$ such that $\langle u\rangle=0, \mathcal{T} u=R$. We have the a priori estimate

$$
\|u\|_{L^{q}\left(\mathbb{T}^{2} \times \mathbb{R}^{2}\right)} \leq \frac{2 \pi}{\left|B_{0,3}\right|}\|R\|_{L^{q}\left(\mathbb{T}^{2} \times \mathbb{R}^{2}\right)}
$$

and if $R \in W^{1, q}\left(\mathbb{T}^{2} \times \mathbb{R}^{2}\right)$ then $u$ belongs to $W^{1, q}\left(\mathbb{T}^{2} \times \mathbb{R}^{2}\right)$ and

$$
\left\|\nabla_{x} u\right\|_{L^{q}\left(\mathbb{T}^{2} \times \mathbb{R}^{2}\right)} \leq \frac{2 \pi}{\left|B_{0,3}\right|}\left\|\nabla_{x} R\right\|_{L^{q}\left(\mathbb{T}^{2} \times \mathbb{R}^{2}\right)}, \quad\left\|\nabla_{p} u\right\|_{L^{q}\left(\mathbb{T}^{2} \times \mathbb{R}^{2}\right)} \leq C\left(B_{0,3}\right)\left\|\nabla_{(x, p)} R\right\|_{L^{q}\left(\mathbb{T}^{2} \times \mathbb{R}^{2}\right)}
$$

for some constant depending on $B_{0,3}$. Moreover, if supp $R \subset \mathbb{T}^{2} \times B_{R_{0}}$, then supp $u \subset$ $\mathbb{T}^{2} \times B_{R_{0}}$, where $B_{R_{0}}=\left\{p \in \mathbb{R}^{2}:|p| \leq R_{0}\right\}$.

iii) If $q=2$ we have the orthogonal decompositions

$$
L^{2}\left(\mathbb{T}^{2} \times \mathbb{R}^{2}\right)=\operatorname{Ker} \mathcal{T} \oplus \operatorname{Range} \mathcal{T}=\operatorname{Ker} \mathcal{T} \oplus \operatorname{Ker}\langle\rangle .
$$

Proof. i) Let us consider a sequence $\left(u_{k}\right)_{k} \subset C_{c}^{1}\left(\mathbb{T}^{2} \times \mathbb{R}^{2}\right)$ such that $\lim _{k \rightarrow+\infty} u_{k}=u$ and $\lim _{k \rightarrow+\infty} \mathcal{T} u_{k}=\mathcal{T} u$ strongly in $L^{q}\left(\mathbb{T}^{2} \times \mathbb{R}^{2}\right)$ if $q \in\left[1,+\infty\left[\right.\right.$ and weakly $\star$ in $L^{\infty}\left(\mathbb{T}^{2} \times \mathbb{R}^{2}\right)$ if $q=+\infty$. Let us denote by $\left(v_{k}\right)_{k}$ the functions

$$
v_{k}(y, p)=u_{k}(x, p), \quad y=x-\frac{{ }^{\perp} p}{B_{0,3}} .
$$

By formula (34) we have

$$
\mathcal{T} u_{k}\left(y+\frac{{ }^{\perp} p}{B_{0,3}}, p\right)=-B_{0,3}{ }^{\perp} p \cdot \nabla_{p} v_{k}(y, p)
$$

and therefore

$$
\begin{aligned}
\left\langle\mathcal{T} u_{k}\right\rangle(y, p) & =\frac{1}{2 \pi} \int_{0}^{2 \pi}\left\langle\mathcal{T} u_{k}\right\rangle\left(y_{1}+\frac{|p| \sin \theta}{B_{0,3}}, y_{2}-\frac{|p| \cos \theta}{B_{0,3}},|p| \cos \theta,|p| \sin \theta\right) d \theta \\
& =-\frac{B_{0,3}}{2 \pi} \int_{0}^{2 \pi}(|p| \sin \theta,-|p| \cos \theta) \cdot \nabla_{p} v_{k}(y,|p| \cos \theta,|p| \sin \theta) d \theta \\
& =\frac{B_{0,3}}{2 \pi} \int_{0}^{2 \pi} \frac{d}{d \theta} v_{k}(y,|p| \cos \theta,|p| \sin \theta) d \theta \\
& =0 .
\end{aligned}
$$

If $q \in\left[1,+\infty\left[\right.\right.$, by using the continuity of the gyro-average in $L^{q}\left(\mathbb{T}^{2} \times \mathbb{R}^{2}\right)$ we obtain

$$
\langle R\rangle=\langle\mathcal{T} u\rangle=\lim _{k \rightarrow+\infty}\left\langle\mathcal{T} u_{k}\right\rangle=0 .
$$


If $q=+\infty$ we have for any $\varphi \in L^{1}\left(\mathbb{T}^{2} \times \mathbb{R}^{2}\right)$, thanks to the weak $\star L^{\infty}\left(\mathbb{T}^{2} \times \mathbb{R}^{2}\right)$ convergence of $\mathcal{T} u_{k}$ towards $\mathcal{T} u$

$$
\begin{aligned}
\int_{\mathbb{T}^{2}} \int_{\mathbb{R}^{2}}\langle R\rangle(y, p) \varphi(y, p) d p d y & =\int_{\mathbb{T}^{2}} \int_{\mathbb{R}^{2}} R(x, p)[\varphi](x, p) d p d x \\
& =\int_{\mathbb{T}^{2}} \int_{\mathbb{R}^{2}} \mathcal{T} u(x, p)[\varphi](x, p) d p d x \\
& =\lim _{k \rightarrow+\infty} \int_{\mathbb{T}^{2}} \int_{\mathbb{R}^{2}} \mathcal{T} u_{k}(x, p)[\varphi](x, p) d p d x \\
& =\lim _{k \rightarrow+\infty} \int_{\mathbb{T}^{2}} \int_{\mathbb{R}^{2}}\left\langle\mathcal{T} u_{k}\right\rangle(y, p) \varphi(y, p) d p d y \\
& =0
\end{aligned}
$$

saying that $\langle R\rangle=0$.

ii) For any $\mu>0$ there is a unique solution $u_{\mu} \in L^{q}\left(\mathbb{T}^{2} \times \mathbb{R}^{2}\right)$ for

$$
\mu u_{\mu}+\mathcal{T} u_{\mu}=R
$$

Indeed, let us denote by $(X, P)(s ; x, p)$ the characteristics associated to $\mathcal{T}$

$$
\frac{d X}{d s}=P(s ; x, p), \quad \frac{d P}{d s}=-B_{0,3}{ }^{\perp} P(s ; x, p), \quad(X, P)(0 ; x, p)=(x, p) .
$$

Since $\operatorname{div}_{(x, p)}\left(p,-B_{0,3}{ }^{\perp} p\right)=0$ the change of variables $(x, p) \rightarrow(X, P)(s ; x, p)$ is measure preserving for any $s$. It is easily seen that

$$
\left(X-\frac{{ }^{\perp} P}{B_{0,3}}\right)(s ; x, p)=x-\frac{{ }^{\perp} p}{B_{0,3}}, \quad P(s ; x, p)=A\left(s B_{0,3}\right) p .
$$

The equation (41) is formally equivalent to

$$
\frac{d}{d s}\left\{e^{\mu s} u_{\mu}(X(s ; x, p), P(s ; x, p))\right\}=e^{\mu s} R(X(s ; x, p), P(s ; x, p))
$$

implying that

$$
u_{\mu}(x, p)=\int_{\mathbb{R}_{-}} e^{\mu s} R(X(s ; x, p), P(s ; x, p)) d s .
$$

We check easily that the function given by (43) belongs to $L^{q}\left(\mathbb{T}^{2} \times \mathbb{R}^{2}\right),\left\|u_{\mu}\right\|_{L^{q}\left(\mathbb{T}^{2} \times \mathbb{R}^{2}\right)} \leq$ $\mu^{-1}\|R\|_{L^{q}\left(\mathbb{T}^{2} \times \mathbb{R}^{2}\right)}$ and solves (41). Moreover, applying the gyro-average operator to (41) we deduce that

$$
\mu\left\langle u_{\mu}\right\rangle=\left\langle\mu u_{\mu}+\mathcal{T} u_{\mu}\right\rangle=\langle R\rangle=0
$$

and thus $\left\langle u_{\mu}\right\rangle=0$ for any $\mu>0$. Actually we can prove that $\left(u_{\mu}\right)_{\mu>0}$ is bounded in $L^{q}\left(\mathbb{T}^{2} \times \mathbb{R}^{2}\right)$. We introduce the function

$$
G(s, x, p)=\int_{s}^{0} R(X(\tau ; x, p), P(\tau ; x, p)) d \tau, \quad(s, x, p) \in \mathbb{R} \times \mathbb{T}^{2} \times \mathbb{R}^{2} .
$$

$\mathrm{RR} \mathrm{n}^{\circ} 0123456789$ 
Notice that $G$ is $\frac{2 \pi}{B_{0,3}}$ periodic with respect to $s$. Indeed we have by (42), (36)

$$
\begin{aligned}
G\left(s-\frac{2 \pi}{B_{0,3}}, x, p\right)-G(s, x, p) & =\int_{s-\frac{2 \pi}{B_{0}, 3}}^{s} R\left(x-\frac{{ }^{\perp} p}{B_{0,3}}+\frac{{ }^{\perp} P(\tau ; x, p)}{B_{0,3}}, P(\tau ; x, p)\right) d \tau \\
& =\frac{1}{B_{0,3}} \int_{s B_{0,3}-2 \pi}^{s B_{0,3}} R\left(x-\frac{{ }^{\perp} p}{B_{0,3}}+\frac{\perp(A(\theta) p)}{B_{0,3}}, A(\theta) p\right) d \theta \\
& =\frac{2 \pi}{B_{0,3}}\langle R\rangle\left(x-\frac{{ }^{\perp} p}{B_{0,3}}, p\right) \\
& =0 .
\end{aligned}
$$

Moreover for any $s \in\left[0, \frac{2 \pi}{\left|B_{0,3}\right|}\right]$ we have

$$
|G(s, x, p)| \leq s\|R\|_{L^{\infty}\left(\mathbb{T}^{2} \times \mathbb{R}^{2}\right)} \leq \frac{2 \pi}{\left|B_{0,3}\right|}\|R\|_{L^{\infty}\left(\mathbb{T}^{2} \times \mathbb{R}^{2}\right)} \text { if } q=+\infty
$$

and if $q \in[1,+\infty[$

$$
\begin{aligned}
\int_{\mathbb{T}^{2}} \int_{\mathbb{R}^{2}}|G(s, x, p)|^{q} d p d x & \leq \int_{\mathbb{T}^{2}} \int_{\mathbb{R}^{2}} s^{q-1} \int_{0}^{s}|R|^{q}(X(\tau ; x, p), P(\tau ; x, p)) d \tau d p d x \\
& =s^{q-1} \int_{0}^{s} \int_{\mathbb{T}^{2}} \int_{\mathbb{R}^{2}}|R|^{q}(X, P) d P d X d \tau \\
& =s^{q}\|R\|_{L^{q}\left(\mathbb{T}^{2} \times \mathbb{R}^{2}\right)} \\
& \leq\left(\frac{2 \pi}{\left|B_{0,3}\right|}\right)^{q}\|R\|_{L^{q}\left(\mathbb{T}^{2} \times \mathbb{R}^{2}\right)}^{q} .
\end{aligned}
$$

Thus for any $q \in[1,+\infty]$ and any $s \in \mathbb{R}$ we have

$$
\|G(s)\|_{L^{q}\left(\mathbb{T}^{2} \times \mathbb{R}^{2}\right)} \leq \frac{2 \pi}{\left|B_{0,3}\right|}\|R\|_{L^{q}\left(\mathbb{T}^{2} \times \mathbb{R}^{2}\right)} .
$$

Now we are ready for estimating $\left\|u_{\mu}\right\|_{L^{q}\left(\mathbb{T}^{2} \times \mathbb{R}^{2}\right)}$ uniformly with respect to $\mu>0$. Observe that

$$
u_{\mu}(x, p)=-\int_{\mathbb{R}_{-}} e^{\mu s} \partial_{s} G(s, x, p) d s=\mu \int_{\mathbb{R}_{-}} e^{\mu s} G(s, x, p) d s
$$

and thus we obtain easily that

$$
\left\|u_{\mu}\right\|_{L^{q}\left(\mathbb{T}^{2} \times \mathbb{R}^{2}\right)} \leq\|G\|_{L^{\infty}\left(\mathbb{R} ; L^{q}\left(\mathbb{T}^{2} \times \mathbb{R}^{2}\right)\right)} \int_{\mathbb{R}_{-}} e^{\mu s} \mu d s \leq \frac{2 \pi}{\left|B_{0,3}\right|}\|R\|_{L^{q}\left(\mathbb{T}^{2} \times \mathbb{R}^{2}\right)} .
$$

Take now a sequence $\left(\mu_{k}\right)_{k}$ converging towards zero such that $\left(u_{\mu_{k}}\right)_{k}$ converges to some function $u \in L^{q}\left(\mathbb{T}^{2} \times \mathbb{R}^{2}\right)$ weakly in $L^{q}\left(\mathbb{T}^{2} \times \mathbb{R}^{2}\right)$ if $q \in[1,+\infty[$, respectively weakly $\star$ in $L^{\infty}\left(\mathbb{T}^{2} \times \mathbb{R}^{2}\right)$ if $q=+\infty$. Notice that

$$
\|u\|_{L^{q}\left(\mathbb{T}^{2} \times \mathbb{R}^{2}\right)} \leq \liminf _{k \rightarrow+\infty}\left\|u_{\mu_{k}}\right\|_{L^{q}\left(\mathbb{T}^{2} \times \mathbb{R}^{2}\right)} \leq \frac{2 \pi}{\left|B_{0,3}\right|}\|R\|_{L^{q}\left(\mathbb{T}^{2} \times \mathbb{R}^{2}\right)} .
$$

INRIA 
Passing to the limit for $k \rightarrow+\infty$ in (41) we obtain that $\mathcal{T} u=R$. Since $\left\langle u_{\mu_{k}}\right\rangle=0$ for any $k$ we have also $\langle u\rangle=0$.

Assume now that $R$ belongs to $W^{1, q}\left(\mathbb{T}^{2} \times \mathbb{R}^{2}\right)$. In order to establish the $W^{1, q}\left(\mathbb{T}^{2} \times \mathbb{R}^{2}\right)$ regularity of $u$ it is sufficient to estimate $u_{\mu}$ in $W^{1, q}\left(\mathbb{T}^{2} \times \mathbb{R}^{2}\right)$ uniformly with respect to $\mu>0$. By 42) notice that

$$
\frac{\partial X}{\partial x}=\mathrm{I}_{2}, \quad \frac{\partial X}{\partial p}=\frac{1}{B_{0,3}} A\left(-\frac{\pi}{2}\right)\left(A\left(s B_{0,3}\right)-\mathrm{I}_{2}\right), \quad \frac{\partial P}{\partial x}=0_{2}, \quad \frac{\partial P}{\partial p}=A\left(s B_{0,3}\right) .
$$

Taking the derivatives with respect to $(x, p)$ in (43) one gets

$$
\begin{aligned}
\nabla_{x} u_{\mu} & =\int_{\mathbb{R}_{-}} e^{\mu s}\left(\frac{\partial X}{\partial x}\right)^{t} \nabla_{x} R(X(s ; x, p), P(s ; x, p)) d s \\
= & \int_{\mathbb{R}_{-}} e^{\mu s} R_{1}(s, x, p) d s \\
\nabla_{p} u_{\mu}= & \int_{\mathbb{R}_{-}} e^{\mu s}\left\{\left(\frac{\partial X}{\partial p}\right)^{t} \nabla_{x} R(X(s), P(s))+\left(\frac{\partial P}{\partial p}\right)^{t} \nabla_{p} R(X(s), P(s))\right\} d s \\
= & \int_{\mathbb{R}_{-}} e^{\mu s} R_{2}(s, x, p) d s
\end{aligned}
$$

with

$$
R_{1}(s, x, p)=\nabla_{x} R(X(s ; x, p), P(s ; x, p))
$$

and

$$
\begin{aligned}
R_{2}(s, x, p) & =\frac{1}{B_{0,3}}\left(A\left(-\frac{\pi}{2}\right)\left(A\left(s B_{0,3}\right)-\mathrm{I}_{2}\right)\right)^{t} \nabla_{x} R(X(s ; x, p), P(s ; x, p)) \\
& +A\left(s B_{0,3}\right)^{t} \nabla_{p} R(X(s ; x, p), P(s ; x, p)) .
\end{aligned}
$$

We introduce the functions $G_{1}, G_{2}$ given by

$$
G_{k}(s, x, p)=\int_{s}^{0} R_{k}(\tau, x, p) d \tau, \quad(s, x, p) \in \mathbb{R} \times \mathbb{T}^{2} \times \mathbb{R}^{2}, \quad k \in\{1,2\} .
$$

Observe that $G_{1}, G_{2}$ are $\frac{2 \pi}{\left|B_{0,3}\right|}$ periodic with respect to $s$. Indeed, by the previous computations we know that

$$
\int_{s-\frac{2 \pi}{B_{0}, 3}}^{s} R(X(\tau ; x, p), P(\tau ; x, p)) d \tau=0, \quad(s, x, p) \in \mathbb{R} \times \mathbb{T}^{2} \times \mathbb{R}^{2}
$$

and thus by taking the derivatives with respect to $(x, p)$ one gets

$$
\int_{s-\frac{2 \pi}{B_{0}, 3}}^{s} R_{k}(\tau, x, p) d \tau=0, \quad k \in\{1,2\}, \quad(s, x, p) \in \mathbb{R} \times \mathbb{T}^{2} \times \mathbb{R}^{2} .
$$

$\mathrm{RR} \mathrm{n}^{\circ} 0123456789$ 
From now on we can proceed exactly as before, when estimating the $L^{q}$ norms of $u_{\mu}$. We have

$$
\begin{gathered}
\left\|G_{1}\right\|_{L^{\infty}\left(\mathbb{R} ; L^{q}\left(\mathbb{T}^{2} \times \mathbb{R}^{2}\right)\right)} \leq \frac{2 \pi}{\left|B_{0,3}\right|}\left\|R_{1}\right\|_{L^{\infty}\left(\mathbb{R} ; L^{q}\left(\mathbb{T}^{2} \times \mathbb{R}^{2}\right)\right)}=\frac{2 \pi}{\left|B_{0,3}\right|}\left\|\nabla_{x} R\right\|_{L^{q}\left(\mathbb{T}^{2} \times \mathbb{R}^{2}\right)} \\
\left\|G_{2}\right\|_{L^{\infty}\left(\mathbb{R} ; L^{q}\left(\mathbb{T}^{2} \times \mathbb{R}^{2}\right)\right)} \leq \frac{2 \pi}{\left|B_{0,3}\right|}\left\|R_{2}\right\|_{L^{\infty}\left(\mathbb{R} ; L^{q}\left(\mathbb{T}^{2} \times \mathbb{R}^{2}\right)\right)} \leq C\left(B_{0,3}\right)\left\|\nabla_{(x, p)} R\right\|_{L^{q}\left(\mathbb{T}^{2} \times \mathbb{R}^{2}\right)}
\end{gathered}
$$

implying that

$$
\left\|\nabla_{x} u_{\mu}\right\|_{L^{q}\left(\mathbb{T}^{2} \times \mathbb{R}^{2}\right)} \leq\left\|G_{1}\right\|_{L^{\infty}\left(\mathbb{R} ; L^{q}\left(\mathbb{T}^{2} \times \mathbb{R}^{2}\right)\right)} \leq \frac{2 \pi}{\left|B_{0,3}\right|}\left\|\nabla_{x} R\right\|_{L^{q}\left(\mathbb{T}^{2} \times \mathbb{R}^{2}\right)}
$$

and

$$
\left\|\nabla_{p} u_{\mu}\right\|_{L^{q}\left(\mathbb{T}^{2} \times \mathbb{R}^{2}\right)} \leq\left\|G_{2}\right\|_{L^{\infty}\left(\mathbb{R} ; L^{q}\left(\mathbb{T}^{2} \times \mathbb{R}^{2}\right)\right)} \leq C\left(B_{0,3}\right)\left\|\nabla_{(x, p)} R\right\|_{L^{q}\left(\mathbb{T}^{2} \times \mathbb{R}^{2}\right)} .
$$

Notice also by (43) that if supp $R \subset \mathbb{T}^{2} \times B_{R_{0}}$ then supp $u_{\mu} \subset \mathbb{T}^{2} \times B_{R_{0}}$ for any $\mu>0$ and therefore the solution $u$ has also compact support in $\mathbb{T}^{2} \times B_{R_{0}}$.

It remains to prove the uniqueness of the solution $u$. Assume that there are $u_{1}, u_{2} \in$ $L^{q}\left(\mathbb{T}^{2} \times \mathbb{R}^{2}\right)$ satisfying

$$
\left\langle u_{1}\right\rangle=\left\langle u_{2}\right\rangle=0, \quad \mathcal{T} u_{1}=\mathcal{T} u_{2}=R .
$$

Since $\mathcal{T}\left(u_{1}-u_{2}\right)=0$ we have by Proposition 3.2

$$
0=\left\langle u_{1}-u_{2}\right\rangle(y, p)=\left(u_{1}-u_{2}\right)\left(y+\frac{{ }^{\perp} p}{B_{0,3}}, p\right), \quad(y, p) \in \mathbb{T}^{2} \times \mathbb{R}^{2}
$$

and thus $u_{1}=u_{2}$.

iii) Assume that $q=2$. The operator $\mathcal{T}$ is densely defined, has closed graph and its adjoint operator is given by $\mathrm{D}\left(\mathcal{T}^{\star}\right)=\mathrm{D}(\mathcal{T}), \mathcal{T}^{\star}=-\mathcal{T}$. By the statements i), ii) we know that Range $\mathcal{T}=\operatorname{Ker}\langle\rangle$ where \langle\rangle denotes the gyro-average operator on $L^{2}\left(\mathbb{T}^{2} \times \mathbb{R}^{2}\right)$. Since \langle\rangle is linear bounded operator on $L^{2}\left(\mathbb{T}^{2} \times \mathbb{R}^{2}\right)$ we deduce that Range $\mathcal{T}$ is closed. It is easily seen that $\operatorname{Ker} \mathcal{T}$ is closed in $L^{2}\left(\mathbb{T}^{2} \times \mathbb{R}^{2}\right)$ and thus we have the orthogonal decomposition

$$
L^{2}\left(\mathbb{T}^{2} \times \mathbb{R}^{2}\right)=\operatorname{Ker} \mathcal{T} \oplus(\operatorname{Ker} \mathcal{T})^{\perp} .
$$

But

$$
(\operatorname{Ker} \mathcal{T})^{\perp}=\left(\operatorname{Ker} \mathcal{T}^{\star}\right)^{\perp}=\overline{\text { Range } \mathcal{T}}=\text { Range } \mathcal{T}=\operatorname{Ker}\langle\rangle
$$

and our conclusion follows.

Based on the properties of the operators $\mathcal{T}$ and \langle\rangle we derive now the limit model for the leading order terms $(f, E)$ in the asymptotic expansions (21), (22). We assume that $(f, E)$ are smooth, such that the equations below are understood in the classical sense. We associate to $f$ the center distribution function $g$ given by

$$
g(t, y, p)=f(t, x, p), \quad y=x-\frac{{ }^{\perp} p}{B_{0,3}} .
$$


By Proposition 3.1 the equation (29) says that $g$ has radial symmetry with respect to $p \in \mathbb{R}^{2}$. Combining (30) and Proposition 3.4 implies

$$
\left\langle\partial_{t} f-E(t, x) \cdot \nabla_{p} f\right\rangle=0 .
$$

Since $\mathcal{T} f=0$ we have by Proposition 3.2

$$
\begin{gathered}
\langle f(t)\rangle(y, p)=f\left(t, y+\frac{{ }^{\perp} p}{B_{0,3}}, p\right) \\
\left\langle E \cdot \nabla_{p} f(t)\right\rangle(y, p)=-\left\langle\frac{{ }^{\perp} E(t)}{B_{0,3}}\right\rangle(y, p) \cdot \nabla_{x} f\left(t, y+\frac{{ }^{\perp} p}{B_{0,3}}, p\right) .
\end{gathered}
$$

Putting together (29), 44), (45), (46) leads to the following equations

$$
\mathcal{T} f=0, \quad \partial_{t} f\left(t, y+\frac{{ }^{\perp} p}{B_{0,3}}, p\right)+\left\langle\frac{{ }^{\perp} E(t)}{B_{0,3}}\right\rangle(y, p) \cdot \nabla_{x} f\left(t, y+\frac{{ }^{\perp} p}{B_{0,3}}, p\right)=0
$$

which is equivalent to

$$
\mathcal{T} f=0, \partial_{t} f(t, x, p)+\left\langle\frac{{ }^{\perp} E(t)}{B_{0,3}}\right\rangle\left(x-\frac{{ }^{\perp} p}{B_{0,3}}, p\right) \cdot \nabla_{x} f(t, x, p)=0 .
$$

Actually it is sufficient to impose the constraint $\mathcal{T} f=0$ only for the initial condition. Indeed, by construction, the function $(y, p) \rightarrow\left\langle\frac{{ }^{\perp} E(t)}{B_{0,3}}\right\rangle(y, p)$ has radial symmetry with respect to $p \in \mathbb{R}^{2}$ and therefore, by Proposition 3.1 the function $(x, p) \rightarrow\left\langle\frac{{ }^{\perp} E(t)}{B_{0,3}}\right\rangle\left(x-\frac{{ }^{\perp} p}{B_{0,3}}, p\right)$ belongs to the kernel of $\mathcal{T}$. Since the operator $\mathcal{T}$ commutes with $\partial_{t}$ and $\nabla_{x}$ we deduce that

$$
\begin{aligned}
\mathcal{T}\left(\partial_{t} f+\left\langle\frac{{ }^{\perp} E(t)}{B_{0,3}}\right\rangle\left(x-\frac{{ }^{\perp} p}{B_{0,3}}, p\right) \cdot \nabla_{x} f\right) & =\partial_{t} \mathcal{T} f+\left\langle\frac{{ }^{\perp} E(t)}{B_{0,3}}\right\rangle\left(x-\frac{{ }^{\perp} p}{B_{0,3}}, p\right) \cdot \nabla_{x} \mathcal{T} f \\
& =0 .
\end{aligned}
$$

Therefore if $\mathcal{T} f(0)=0$ then $\mathcal{T} f(t)=0$ for any $t \in \mathbb{R}_{+}$. It remains to add the equations for the electric field, cf. (26). Therefore the limit model is

$$
\begin{cases}\partial_{t} f+\left\langle\frac{{ }^{\perp} E(t)}{B_{0,3}}\right\rangle\left(x-\frac{\perp p}{B_{0,3}}, p\right) \cdot \nabla_{x} f=0, & (t, x, p) \in \mathbb{R}_{+} \times \mathbb{T}^{2} \times \mathbb{R}^{2} \\ \mathcal{T} f(0)=0, & (x, p) \in \mathbb{T}^{2} \times \mathbb{R}^{2} \\ \operatorname{div}_{x} E=1-\int_{\mathbb{R}^{2}} f d p, \operatorname{div}_{x} \perp^{\perp}=0, & (t, x) \in \mathbb{R}_{+} \times \mathbb{T}^{2} .\end{cases}
$$

Since $\operatorname{div}_{x}{ }^{\perp} E=0$ it is easily seen that $\operatorname{div}_{x}\left\langle\frac{{ }^{\perp} E(t)}{B_{0,3}}\right\rangle\left(x-\frac{{ }^{\perp} p}{B_{0,3}}, p\right)=0$ and thus the transport equation of the above model can be written also in the conservative form

$$
\partial_{t} f+\operatorname{div}_{x}\left(\left\langle\frac{{ }^{\perp} E(t)}{B_{0,3}}\right\rangle\left(x-\frac{{ }^{\perp} p}{B_{0,3}}, p\right) f\right)=0, \quad(t, x, p) \in \mathbb{R}_{+} \times \mathbb{T}^{2} \times \mathbb{R}^{2} .
$$

Remark 3.2 If the density $f$ satisfies (29), (30) in the sense of distributions, we obtain the same limit model which, in this case, has to be understood in the sense of distributions too.

RR $\quad n^{\circ} 0123456789$ 


\section{Well-posedness of the limit model}

In this section we construct strong solutions for the limit model (47). We only sketch the main arguments, the other details being left to the reader. We indicate how to get a priori bounds for the solution $(f, E)$, which, after standard manipulations, will allow us to construct strong solutions, at least locally in time. Actually, employing similar techniques as those used for the Vlasov-Poisson system it is possible to construct global in time strong solutions cf. [30, 27, 28, 3, but we do not go further into these directions. In the sequel the notation $C$ stands for various constants depending on the initial conditions, which can change from line to line.

We assume that the initial condition $f_{0}$ satisfies the hypotheses

H4) $f_{0} \geq 0, \quad \int_{\mathbb{T}^{2}} \int_{\mathbb{R}^{2}} f_{0}(x, p) d p d x=1$;

H5) $f_{0}, \nabla_{x} f_{0} \in L^{q}\left(\mathbb{T}^{2} \times \mathbb{R}^{2}\right)$ for some $\left.\left.q \in\right] 2,+\infty\right], \mathcal{T} f_{0}=0$;

H6) supp $f_{0} \subset \mathbb{T}^{2} \times B_{R}$ for some $R>0$, where $B_{R}=\left\{p \in \mathbb{R}^{2}:|p| \leq R\right\}$.

For any smooth field $E \in L^{\infty}(] 0, T\left[; W^{1, \infty}\left(\mathbb{T}^{2}\right)\right)$ we associate the characteristics $X=$ $X(s ; t, x, p)$ given by

$$
\left\{\begin{array}{l}
\frac{d}{d s} X(s ; t, x, p)=\mathcal{V}_{E}\left(s, X(s ; t, x, p)-\frac{\perp p}{B_{0,3}}, p\right), \quad s \in[0, T] \\
X(t ; t, x, p)=x
\end{array}\right.
$$

with the notation $\mathcal{V}_{E}(t, y, p)=\left\langle\frac{{ }^{\perp} E(t)}{B_{0,3}}\right\rangle(y, p), \quad(y, p) \in \mathbb{T}^{2} \times \mathbb{R}^{2}$. Notice that $\mathcal{V}_{E}$ is also smooth with respect to $(y, p)$ and we have

$$
\left\|\frac{\partial \mathcal{V}_{E}(t)}{\partial(y, p)}\right\|_{L^{\infty}\left(\mathbb{T}^{2} \times \mathbb{R}^{2}\right)} \leq C\left\|\frac{\partial E(t)}{\partial x}\right\|_{L^{\infty}\left(\mathbb{T}^{2}\right)}, \quad t \in[0, T] .
$$

Therefore the characteristics in (49) are well defined for any $(t, x, p) \in[0, T] \times \mathbb{T}^{2} \times \mathbb{R}^{2}$ and there are smooth with respect to $(x, p)$. The solution of the transport equation in (47) is given by

$$
f(t, x, p)=f_{0}(X(0 ; t, x, p), p), \quad(t, x, p) \in[0, T] \times \mathbb{T}^{2} \times \mathbb{R}^{2} .
$$

Since $\operatorname{div}_{y} \mathcal{V}_{E}=0$ we have $\left|\operatorname{det}\left(\frac{\partial X}{\partial x}\right)\right|=1$ and thus

$$
\int_{\mathbb{T}^{2}} \int_{\mathbb{R}^{2}}|f(t, x, p)|^{q} d p d x=\int_{\mathbb{T}^{2}} \int_{\mathbb{R}^{2}}\left|f_{0}(x, p)\right|^{q} d p d x, \quad t \in[0, T] .
$$

By the hypothesis H6 we deduce that $\operatorname{supp} f(t) \subset \mathbb{T}^{2} \times B_{R}$ for any $t$. By Hölder inequality we have, with the notation $\rho=\int_{\mathbb{R}^{2}} f d p$ and $1 / q+1 / q^{\prime}=1$

$$
\int_{\mathbb{T}^{2}}|\rho(t, x)|^{q} d x \leq \int_{\mathbb{T}^{2}} \int_{\mathbb{R}^{2}}|f(t, x, p)|^{q} d p d x\left(\pi R^{2}\right)^{q / q^{\prime}}
$$


implying that

$$
\|\rho(t)\|_{L^{q}\left(\mathbb{T}^{2}\right)} \leq\left(\pi R^{2}\right)^{1 / q^{\prime}}\|f(t)\|_{L^{q}\left(\mathbb{T}^{2} \times \mathbb{R}^{2}\right)}=\left(\pi R^{2}\right)^{1 / q^{\prime}}\left\|f_{0}\right\|_{L^{q}\left(\mathbb{T}^{2} \times \mathbb{R}^{2}\right)} .
$$

By elliptic regularity results and Sobolev inequalities we deduce the following bound for the electric field

$$
\begin{aligned}
\|E(t)\|_{L^{\infty}\left(\mathbb{T}^{2}\right)} & \leq C\|E(t)\|_{W^{1, q}\left(\mathbb{T}^{2}\right)} \leq C\left(1+\|\rho(t)\|_{L^{q}\left(\mathbb{T}^{2}\right)}\right) \\
& \leq C\left(1+\left(\pi R^{2}\right)^{1 / q^{\prime}}\left\|f_{0}\right\|_{L^{q}\left(\mathbb{T}^{2} \times \mathbb{R}^{2}\right)}\right) .
\end{aligned}
$$

Similarly one gets

$$
\begin{aligned}
\left\|\frac{\partial E(t)}{\partial x}\right\|_{L^{\infty}\left(\mathbb{T}^{2}\right)} & \leq C\left\|\frac{\partial E(t)}{\partial x}\right\|_{W^{1, q}\left(\mathbb{T}^{2}\right)} \leq C\left\|\nabla_{x} \rho(t)\right\|_{L^{q}\left(\mathbb{T}^{2}\right)} \\
& \leq C\left(\pi R^{2}\right)^{1 / q^{\prime}}\left\|\nabla_{x} f(t)\right\|_{L^{q}\left(\mathbb{T}^{2} \times \mathbb{R}^{2}\right)} .
\end{aligned}
$$

In order to estimate the norm $\left\|\nabla_{x} f(t)\right\|_{L^{q}\left(\mathbb{T}^{2} \times \mathbb{R}^{2}\right)}$ we multiply the transport equation

$$
\partial_{t} \nabla_{x} f+\left(\mathcal{V}_{E} \cdot \nabla_{x}\right) \nabla_{x} f=-\left(\frac{\partial \mathcal{V}_{E}}{\partial x}\right)^{t} \nabla_{x} f
$$

by $\nabla_{x} f\left|\nabla_{x} f\right|^{q-2}$ and after standard computations one gets

$$
\frac{1}{q} \frac{d}{d t} \int_{\mathbb{T}^{2}} \int_{\mathbb{R}^{2}}\left|\nabla_{x} f(t, x, p)\right|^{q} d p d x \leq \frac{1}{\left|B_{0,3}\right|}\left\|\frac{\partial E(t)}{\partial x}\right\|_{L^{\infty}\left(\mathbb{T}^{2}\right)} \int_{\mathbb{T}^{2}} \int_{\mathbb{R}^{2}}\left|\nabla_{x} f(t, x, p)\right|^{q} d p d x .
$$

Applying Gronwall lemma yields

$$
\left\|\nabla_{x} f(t)\right\|_{L^{q}\left(\mathbb{T}^{2} \times \mathbb{R}^{2}\right)} \leq\left\|\nabla_{x} f_{0}\right\|_{L^{q}\left(\mathbb{T}^{2} \times \mathbb{R}^{2}\right)} \exp \left(\frac{1}{\left|B_{0,3}\right|} \int_{0}^{t}\left\|\frac{\partial E(s)}{\partial x}\right\|_{L^{\infty}\left(\mathbb{T}^{2}\right)} d s\right) .
$$

Combining (50), (51) we deduce that

$$
\left\|\frac{\partial E(t)}{\partial x}\right\|_{L^{\infty}\left(\mathbb{T}^{2}\right)} \leq C \exp \left(\frac{1}{\left|B_{0,3}\right|} \int_{0}^{t}\left\|\frac{\partial E(s)}{\partial x}\right\|_{L^{\infty}\left(\mathbb{T}^{2}\right)} d s\right)
$$

which provides a local in time bound for $\left\|\frac{\partial E}{\partial x}\right\|_{L^{\infty}\left(\mathbb{T}^{2}\right)}$ and also for $\left\|\nabla_{x} f\right\|_{L^{q}\left(\mathbb{T}^{2} \times \mathbb{R}^{2}\right)}$, cf. (51) and $\left\|\partial_{t} f\right\|_{L^{q}\left(\mathbb{T}^{2} \times \mathbb{R}^{2}\right)}=\left\|\mathcal{V}_{E} \cdot \nabla_{x} f\right\|_{L^{q}\left(\mathbb{T}^{2} \times \mathbb{R}^{2}\right)}$. The estimate for $\partial_{t} E$ follows by taking the time derivative of the Poisson equation

$$
\operatorname{div}_{x} \partial_{t} E=-\partial_{t} \rho, \operatorname{div}_{x} \partial_{t}^{\perp} E=0
$$

and by elliptic regularity results $\left\|\partial_{t} E(t)\right\|_{L^{\infty}\left(\mathbb{T}^{2}\right)} \leq C\left\|\partial_{t} E(t)\right\|_{W^{1, q}\left(\mathbb{T}^{2}\right)} \leq C\left\|\partial_{t} \rho(t)\right\|_{L^{q}\left(\mathbb{T}^{2}\right)} \leq C\left(\pi R^{2}\right)^{1 / q^{\prime}}\left\|\partial_{t} f(t)\right\|_{L^{q}\left(\mathbb{T}^{2} \times \mathbb{R}^{2}\right)}$.

Based on these a priori estimates we establish the following result.

$\mathrm{RR} \quad \mathrm{n}^{\circ} 0123456789$ 
Proposition 4.1 Assume that the initial condition $f_{0}$ satisfies the hypotheses $\mathrm{H}_{4}, \mathrm{H} 5 \mathrm{H}$ H. Then there is $T>0$ and a local in time strong solution $(f, E)$ on $[0, T]$ for the limit model (47). The solution is unique and satisfies

$$
\begin{gathered}
f \geq 0, \quad \int_{\mathbb{T}^{2}} \int_{\mathbb{R}^{2}} f(t, x, p) d p d x=1, \quad t \in[0, T] \\
f, \partial_{t} f, \nabla_{x} f \in L^{\infty}(] 0, T\left[; L^{q}\left(\mathbb{T}^{2} \times \mathbb{R}^{2}\right)\right), \operatorname{supp} f(t) \subset \mathbb{T}^{2} \times B_{R}, \quad t \in[0, T] \\
E \in W^{1, \infty}(] 0, T\left[\times \mathbb{T}^{2}\right), \quad \int_{\mathbb{T}^{2}}|E(t, x)|^{2} d x=\int_{\mathbb{T}^{2}}\left|E_{0}(x)\right|^{2} d x, \quad t \in[0, T] .
\end{gathered}
$$

Moreover if $\nabla_{p} f_{0} \in L^{q}\left(\mathbb{T}^{2} \times \mathbb{R}^{2}\right)$ then $\nabla_{p} f \in L^{\infty}(] 0, T\left[; L^{q}\left(\mathbb{T}^{2} \times \mathbb{R}^{2}\right)\right)$ and if the initial kinetic energy is finite $\int_{\mathbb{T}^{2}} \int_{\mathbb{R}^{2}} \frac{|p|^{2}}{2} f_{0}(x, p) d p d x<+\infty$ then the kinetic energy is conserved too

$$
\int_{\mathbb{T}^{2}} \int_{\mathbb{R}^{2}} \frac{|p|^{2}}{2} f(t, x, p) d p d x=\int_{\mathbb{T}^{2}} \int_{\mathbb{R}^{2}} \frac{|p|^{2}}{2} f_{0}(x, p) d p d x, \quad t \in[0, T] .
$$

Proof. We justify only the conservations of the electric and kinetic energies, the estimate for $\nabla_{p} f$ and the uniqueness of the strong solution. Using the continuity equation

$$
\partial_{t} \int_{\mathbb{R}^{2}} f d p+\operatorname{div}_{x} \int_{\mathbb{R}^{2}} \mathcal{V}_{E} f d p=0
$$

we obtain easily that

$$
\begin{aligned}
\frac{1}{2} \frac{d}{d t} \int_{\mathbb{T}^{2}}|E(t, x)|^{2} d x & =\int_{\mathbb{T}^{2}} E(t, x) \int_{\mathbb{R}^{2}} \mathcal{V}_{E}\left(t, x-\frac{{ }^{\perp} p}{B_{0,3}}, p\right) f(t, x, p) d p d x \\
& =\int_{\mathbb{T}^{2}} \int_{\mathbb{R}^{2}} E\left(t, y+\frac{{ }^{\perp} p}{B_{0,3}}\right) \mathcal{V}_{E}(t, y, p) g(t, y, p) d p d y
\end{aligned}
$$

where $g(t, y, p)=f(t, x, p), y=x-\frac{\perp}{B_{0,3}}$. Recall that $\mathcal{V}_{E}(t, y, p)$ and $g(t, y, p)$ have radial symmetry with respect to $p \in \mathbb{R}^{2}$ and thus we can write

$$
\begin{aligned}
\frac{1}{2} \frac{d}{d t} \int_{\mathbb{T}^{2}}|E|^{2} d x & =\int_{\mathbb{T}^{2}} \int_{\mathbb{R}_{+}}\left(\mathcal{V}_{E} g\right)(t, y, r) r \int_{0}^{2 \pi} E\left(t, y_{1}+\frac{r \sin \theta}{B_{0,3}}, y_{2}-\frac{r \cos \theta}{B_{0,3}}\right) d \theta d r d y \\
& =-B_{0,3} \int_{\mathbb{T}^{2}} \int_{\mathbb{R}^{2}} g(t, y, p) \mathcal{V}_{E}(t, y, p)^{\perp} \mathcal{V}_{E}(t, y, p) d p d y=0
\end{aligned}
$$

The conservation of the kinetic energy follows immediately by integrating with respect to $(x, p)$ the equation

$$
\partial_{t}\left(\frac{|p|^{2}}{2} f\right)+\operatorname{div}_{x}\left(\frac{|p|^{2}}{2} \mathcal{V}_{E} f\right)=0
$$


Assume now that $\nabla_{p} f_{0}$ belongs to $L^{q}\left(\mathbb{T}^{2} \times \mathbb{R}^{2}\right)$. Multiplying the transport equation

$$
\partial_{t} \nabla_{p} f+\left(\mathcal{V}_{E} \cdot \nabla_{x}\right) \nabla_{p} f=-\left(\frac{\partial\left\{\mathcal{V}_{E}\left(t, x-\frac{{ }^{\perp} p}{B_{0,3}}, p\right)\right\}}{\partial p}\right)^{t} \nabla_{p} f
$$

by $\nabla_{p} f\left|\nabla_{p} f\right|^{q-2}$ yields after usual manipulations

$$
\frac{1}{q} \frac{d}{d t} \int_{\mathbb{T}^{2}} \int_{\mathbb{R}^{2}}\left|\nabla_{p} f(t, x, p)\right|^{q} d p d x \leq C\left\|\frac{\partial E(t)}{\partial x}\right\|_{L^{\infty}\left(\mathbb{T}^{2}\right)} \int_{\mathbb{T}^{2}} \int_{\mathbb{R}^{2}}\left|\nabla_{p} f(t, x, p)\right|^{q} d p d x .
$$

Applying Gronwall lemma yields

$$
\left\|\nabla_{p} f(t)\right\|_{L^{q}\left(\mathbb{T}^{2} \times \mathbb{R}^{2}\right)} \leq\left\|\nabla_{p} f_{0}\right\|_{L^{q}\left(\mathbb{T}^{2} \times \mathbb{R}^{2}\right)} \exp \left(C \int_{0}^{t}\left\|\frac{\partial E(s)}{\partial x}\right\|_{L^{\infty}\left(\mathbb{T}^{2}\right)} d s\right), \quad t \in[0, T] .
$$

Assume now that $\left(f_{1}, E_{1}\right),\left(f_{2}, E_{2}\right)$ are two smooth solutions on $[0, T]$ verifying $f_{1}(0)=$ $f_{2}(0)=f_{0}$. Since $f_{1}, f_{2} \in L^{\infty}(] 0, T\left[; L^{q}\left(\mathbb{T}^{2} \times \mathbb{R}^{2}\right)\right)$ are compactly supported, $q>2$, we have also $f_{1}, f_{2} \in L^{\infty}(] 0, T\left[; L^{2}\left(\mathbb{T}^{2} \times \mathbb{R}^{2}\right)\right)$ and

$$
\frac{d}{d t} \int_{\mathbb{T}^{2}} \int_{\mathbb{R}^{2}}\left|f_{1}(t, x, p)\right|^{2} d p d x=\frac{d}{d t} \int_{\mathbb{T}^{2}} \int_{\mathbb{R}^{2}}\left|f_{2}(t, x, p)\right|^{2} d p d x=0 .
$$

Multiplying by $f_{2}$ the equation

$$
\partial_{t} f_{1}+\mathcal{V}_{2} \cdot \nabla_{x} f_{1}=\left(\mathcal{V}_{2}-\mathcal{V}_{1}\right) \cdot \nabla_{x} f_{1}
$$

we obtain

$\frac{d}{d t} \int_{\mathbb{T}^{2}} \int_{\mathbb{R}^{2}} f_{1} f_{2} d p d x-\int_{\mathbb{T}^{2}} \int_{\mathbb{R}^{2}} f_{1}\left(\partial_{t} f_{2}+\mathcal{V}_{2} \cdot \nabla_{x} f_{2}\right) d p d x=\int_{\mathbb{T}^{2}} \int_{\mathbb{R}^{2}} f_{2}\left(\mathcal{V}_{2}-\mathcal{V}_{1}\right) \cdot \nabla_{x} f_{1} d p d x$

Since $\operatorname{div}_{x}\left(\mathcal{V}_{2}-\mathcal{V}_{1}\right)=0$ we have $\int_{\mathbb{T}^{2}} \int_{\mathbb{R}^{2}} f_{1}\left(\mathcal{V}_{2}-\mathcal{V}_{1}\right) \cdot \nabla_{x} f_{1} d p d x=0$ and thus the previous equality can be written

$$
\begin{aligned}
\frac{1}{2} \frac{d}{d t} \int_{\mathbb{T}^{2}} \int_{\mathbb{R}^{2}}\left|f_{1}-f_{2}\right|^{2} d p d x & =-\frac{d}{d t} \int_{\mathbb{T}^{2}} \int_{\mathbb{R}^{2}} f_{1} f_{2} d p d x \\
& =-\int_{\mathbb{T}^{2}} \int_{\mathbb{R}^{2}}\left(f_{1}-f_{2}\right)\left(\mathcal{V}_{1}-\mathcal{V}_{2}\right) \cdot \nabla_{x} f_{1} \mathbf{1}_{\{|p| \leq R\}} d p d x \\
& \leq\left\|\left(f_{1}-f_{2}\right)(t)\right\|_{L^{2}\left(\mathbb{T}^{2} \times \mathbb{R}^{2}\right)}\left\|\mathcal{V}_{1}-\mathcal{V}_{2}\right\|_{L^{l}\left(\mathbb{T}^{2} \times B_{R}\right)}\left\|\nabla_{x} f_{1}\right\|_{L^{q}\left(\mathbb{T}^{2} \times \mathbb{R}^{2}\right)}
\end{aligned}
$$

with $1 / l+1 / q=1 / 2$. A direct computation shows that

$$
\left\|\left(\mathcal{V}_{1}-\mathcal{V}_{2}\right)(t)\right\|_{L^{l}\left(\mathbb{T}^{2} \times B_{R}\right)} \leq \frac{\left(\pi R^{2}\right)^{1 / l}}{\left|B_{0,3}\right|}\left\|\left(E_{1}-E_{2}\right)(t)\right\|_{L^{l}\left(\mathbb{T}^{2}\right)}
$$

RR $\quad n^{\circ} 0123456789$ 
Combining now the Poisson equations

$$
\operatorname{div}_{x}\left(E_{1}-E_{2}\right)=-\int_{\mathbb{R}^{2}}\left(f_{1}-f_{2}\right) d p=-\left(\rho_{1}-\rho_{2}\right), \operatorname{div}_{x}{ }^{\perp}\left(E_{1}-E_{2}\right)=0
$$

one gets by Sobolev inequality

$$
\left\|\left(E_{1}-E_{2}\right)(t)\right\|_{L^{l}\left(\mathbb{T}^{2}\right)} \leq C\left\|\rho_{1}-\rho_{2}\right\|_{L^{2}\left(\mathbb{T}^{2}\right)} \leq C\left(\pi R^{2}\right)^{1 / 2}\left\|\left(f_{1}-f_{2}\right)(t)\right\|_{L^{2}\left(\mathbb{T}^{2} \times \mathbb{R}^{2}\right)} .
$$

Putting together the inequalities (53), (54), (56) yields

$$
\int_{\mathbb{T}^{2}} \int_{\mathbb{R}^{2}}\left|\left(f_{1}-f_{2}\right)(t, x, p)\right|^{2} d p d x \leq C \int_{0}^{t} \int_{\mathbb{T}^{2}} \int_{\mathbb{R}^{2}}\left|\left(f_{1}-f_{2}\right)(s, x, p)\right|^{2} d p d x d s, \quad t \in[0, T]
$$

saying that $f_{1}(t)=f_{2}(t)$ for any $t \in[0, T]$. Coming back in (56) we deduce also that $E_{1}(t)=E_{2}(t)$ for any $t \in[0, T]$.

By similar arguments we can prove further regularity results for the strong solution of the limit model (47). The proof is standard and is left to the reader.

Proposition 4.2 Assume that the initial condition $f_{0}$ belongs to $W^{2, q}\left(\mathbb{T}^{2} \times \mathbb{R}^{2}\right)$ for some $q \in] 2,+\infty]$ and verifies $\mathcal{T} f_{0}=0$ and the hypotheses $H_{4}, H 6$. Then the local in time strong solution $(f, E)$ constructed in Proposition 4.1 satisfies

$$
\nabla_{(t, x, p)}^{2} f \in L^{\infty}(] 0, T\left[; L^{q}\left(\mathbb{T}^{2} \times \mathbb{R}^{2}\right)\right), \quad E \in W^{2, \infty}(] 0, T\left[\times \mathbb{T}^{2}\right) .
$$

\section{Convergence results}

We justify now the convergence of the solutions $\left(f^{\varepsilon}, E^{\varepsilon}\right)_{\varepsilon>0}$ for the Vlasov-Poisson system (9), (10), (11) towards the solution $(f, E)$ for the limit problem (47) when $\varepsilon$ goes to zero. We assume that the initial conditions $\left(f_{0}^{\varepsilon}\right)_{\varepsilon>0}$ satisfy $\mathrm{H} 1$ and $\mathrm{H} 3$ with $F_{0}$ compactly supported in $[0, R]$ for some $R>0$. Notice that in this case $\mathrm{H} 2$ is automatically verified. Moreover we suppose that

H7) $\sup _{\varepsilon>0}\left\|f_{0}^{\varepsilon}\right\|_{W^{2, q}\left(\mathbb{T}^{2} \times \mathbb{R}^{2}\right)}<+\infty$ for some $\left.\left.q \in\right] 2,+\infty\right]$.

Thanks to the compact inclusion $W^{2, q}\left(\mathbb{T}^{2} \times B_{R}\right) \hookrightarrow L^{2}\left(\mathbb{T}^{2} \times B_{R}\right)$ one gets the convergence

$$
\lim _{k \rightarrow+\infty} f_{0}^{\varepsilon_{k}}=f_{0}, \text { strongly in } L^{2}\left(\mathbb{T}^{2} \times \mathbb{R}^{2}\right)
$$

for some sequence $\left(\varepsilon_{k}\right)_{k}$ converging towards zero and some function $f_{0}$ satisfying

$$
f_{0} \geq 0, \quad \int_{\mathbb{T}^{2}} \int_{\mathbb{R}^{2}} f_{0} d p d x=1, \quad f_{0} \in W^{2, q}\left(\mathbb{T}^{2} \times \mathbb{R}^{2}\right), \quad \operatorname{supp} f_{0} \subset \mathbb{T}^{2} \times B_{R} .
$$

INRIA 
Moreover we suppose that $f_{0}$ belongs to the kernel of the operator $\mathcal{T}$ and thus, by Proposition 4.1 there is $T>0$ and a local in time strong solution $(f, E)$ on $[0, T]$ for the limit model (47). We intend to prove the convergence of $\left(f^{\varepsilon_{k}}, E^{\varepsilon_{k}}\right)_{k}$ towards $(f, E)$ in $L^{\infty}(] 0, T\left[; L^{2}\left(\mathbb{T}^{2} \times\right.\right.$ $\left.\left.\mathbb{R}^{2}\right)\right) \times L^{\infty}(] 0, T\left[; L^{2}\left(\mathbb{T}^{2}\right)\right)$.

Theorem 5.1 Assume that the initial conditions $\left(f_{0}^{\varepsilon}\right)_{\varepsilon>0}$ satisfy $H 1$, H3 (with supp $F_{0} \subset$ $[0, R]$ for some $R>0), H^{7}$ and have a limit point $f_{0} \in L^{2}\left(\mathbb{T}^{2} \times \mathbb{R}^{2}\right)$ in the kernel of the operator $\mathcal{T}$

$$
\exists\left(\varepsilon_{k}\right)_{k}, \quad \lim _{k \rightarrow+\infty} \varepsilon_{k}=0, \lim _{k \rightarrow+\infty} f_{0}^{\varepsilon_{k}}=f_{0} \text { strongly in } L^{2}\left(\mathbb{T}^{2} \times \mathbb{R}^{2}\right), \quad \mathcal{T} f_{0}=0 .
$$

Let us denote by $(f, E)$ the local in time solution of the limit model (47). Then the solutions $\left(f^{\varepsilon_{k}}, E^{\varepsilon_{k}}\right)_{k}$ of the Vlasov-Poisson system (9), (10), 11) with $\varepsilon=\varepsilon_{k}$ converge towards $(f, E)$ strongly in $L^{\infty}(] 0, T\left[; L^{2}\left(\mathbb{T}^{2} \times \mathbb{R}^{2}\right)\right) \times L^{\infty}(] 0, T\left[; L^{2}\left(\mathbb{T}^{2}\right)\right)$ and there is a constant $C_{T}$ depending on the initial conditions and $T$ such that we have for any $t \in[0, T], \varepsilon>0$

$$
\left\|\left(f^{\varepsilon}-f\right)(t)\right\|_{L^{2}\left(\mathbb{T}^{2} \times \mathbb{R}^{2}\right)}+\left\|\left(E^{\varepsilon}-E\right)(t)\right\|_{L^{2}\left(\mathbb{T}^{2}\right)} \leq C_{T}\left(\left\|f_{0}^{\varepsilon}-f_{0}\right\|_{L^{2}\left(\mathbb{T}^{2} \times \mathbb{R}^{2}\right)}+\varepsilon^{2}\right) .
$$

Proof. Observe that the gyro-average of $\partial_{t} f-E(t, x) \cdot \nabla_{p} f$ vanishes. Indeed, by Proposition 3.2 we have

$$
\begin{aligned}
\left\langle\partial_{t} f\right. & \left.-E(t, x) \cdot \nabla_{p} f\right\rangle(y, p)=\partial_{t}\langle f\rangle(y, p)-\left\langle E \cdot \nabla_{p} f\right\rangle(y, p) \\
& =\partial_{t} f\left(t, y+\frac{{ }^{\perp} p}{B_{0,3}}, p\right)+\left\langle\frac{{ }^{\perp} E(t)}{B_{0,3}}\right\rangle(y, p) \cdot \nabla_{x} f\left(t, y+\frac{{ }^{\perp} p}{B_{0,3}}, p\right)=0 .
\end{aligned}
$$

Therefore, Proposition 3.4 implies that $\partial_{t} f-E(t, x) \cdot \nabla_{p} f \in \operatorname{Range} \mathcal{T}$ for any $t \in[0, T]$. Let $u$ such that

$$
\partial_{t} f-E(t, x) \cdot \nabla_{p} f+\mathcal{T} u=0,\langle u(t)\rangle=0, \quad t \in[0, T] .
$$

The regularity of the solution $(f, E)$ (cf. Proposition 4.2) implies that $\partial_{t} f-E(t, x) \cdot \nabla_{p} f \in$ $L^{\infty}(] 0, T\left[; W^{1, q}\left(\mathbb{T}^{2} \times \mathbb{R}^{2}\right)\right)$ and thus, thanks to the regularity result in Proposition 3.4 we deduce that $u \in L^{\infty}(] 0, T\left[; W^{1, q}\left(\mathbb{T}^{2} \times \mathbb{R}^{2}\right)\right)$. Moreover we have

$$
\operatorname{supp}\left(\partial_{t} f-E(t, x) \cdot \nabla_{p} f\right) \subset \mathbb{T}^{2} \times B_{R}
$$

implying that $\operatorname{supp} u(t) \subset \mathbb{T}^{2} \times B_{R}$ for any $t \in[0, T]$. Observe also that

$$
\mathcal{T} \partial_{t} u=-\left(\partial_{t}^{2} f-\partial_{t} E \cdot \nabla_{p} f-E \cdot \nabla_{p} \partial_{t} f\right) \in L^{\infty}(] 0, T\left[; L^{q}\left(\mathbb{T}^{2} \times \mathbb{R}^{2}\right)\right), \quad\left\langle\partial_{t} u(t)\right\rangle=0
$$

and therefore applying again the regularity result in Proposition 3.4 one gets $\partial_{t} u \in L^{\infty}(] 0, T\left[; L^{q}\left(\mathbb{T}^{2} \times\right.\right.$ $\left.\mathbb{R}^{2}\right)$ ). Multiplying by $f^{\varepsilon}-f-\varepsilon^{2} u$ the transport equation

$$
\left(\partial_{t}+\varepsilon^{-2} \mathcal{T}-E^{\varepsilon} \cdot \nabla_{p}\right)\left(f^{\varepsilon}-f-\varepsilon^{2} u\right)=-\varepsilon^{2} \partial_{t} u+\varepsilon^{2} E^{\varepsilon} \cdot \nabla_{p} u+\left(E^{\varepsilon}-E\right) \cdot \nabla_{p} f
$$

RR $n^{\circ} 0123456789$ 
one gets after integration with respect to $(x, p) \in \mathbb{T}^{2} \times \mathbb{R}^{2}$

$$
\begin{aligned}
\frac{1}{2} \frac{d}{d t} \int_{\mathbb{T}^{2}} \int_{\mathbb{R}^{2}}\left|f^{\varepsilon}-f-\varepsilon^{2} u\right|^{2} d p d x & =\varepsilon^{2} \int_{\mathbb{T}^{2}} \int_{\mathbb{R}^{2}}\left(-\partial_{t} u+E^{\varepsilon} \cdot \nabla_{p} u\right)\left(f^{\varepsilon}-f-\varepsilon^{2} u\right) d p d x \\
& +\int_{\mathbb{T}^{2}} \int_{\mathbb{R}^{2}}\left(f^{\varepsilon}-f-\varepsilon^{2} u\right)\left(E^{\varepsilon}-E\right) \cdot \nabla_{p} f d p d x \\
& \leq C\left(\varepsilon^{4}+\int_{\mathbb{T}^{2}} \int_{\mathbb{R}^{2}}\left|f^{\varepsilon}-f-\varepsilon^{2} u\right|^{2} d p d x\right) \\
& +C\left\|f^{\varepsilon}-f\right\|_{L^{2}\left(\mathbb{T}^{2} \times \mathbb{R}^{2}\right)}\left\|f^{\varepsilon}-f-\varepsilon^{2} u\right\|_{L^{2}\left(\mathbb{T}^{2} \times \mathbb{R}^{2}\right) .}
\end{aligned}
$$

In the last line of the above estimate we have used that $\partial_{t} u, \nabla_{p} u \in L^{\infty}(] 0, T\left[; L^{2}\left(\mathbb{T}^{2} \times \mathbb{R}^{2}\right)\right)$, that $\left(E^{\varepsilon}\right)_{\varepsilon>0}$ are uniformly bounded in $L^{\infty}(] 0, T\left[\times \mathbb{T}^{2}\right)$ (cf. Proposition [2.1) and that for $1 / l+1 / q=1 / 2$ the following inequalities hold

$$
\begin{aligned}
\mid \int_{\mathbb{T}^{2}} \int_{\mathbb{R}^{2}}\left(f^{\varepsilon}-f-\varepsilon^{2} u\right) & \left(E^{\varepsilon}-E\right) \cdot \nabla_{p} f d p d x \mid \leq\left\|f^{\varepsilon}-f-\varepsilon^{2} u\right\|_{L^{2}\left(\mathbb{T}^{2} \times \mathbb{R}^{2}\right)} \\
& \times\left\|\nabla_{p} f\right\|_{L^{q}\left(\mathbb{T}^{2} \times \mathbb{R}^{2}\right)}\left\|E^{\varepsilon}-E\right\|_{L^{l}\left(\mathbb{T}^{2} \times B_{R}\right)} \\
\leq & C\left\|f^{\varepsilon}-f-\varepsilon^{2} u\right\|_{L^{2}\left(\mathbb{T}^{2} \times \mathbb{R}^{2}\right)}\left\|\rho^{\varepsilon}-\rho\right\|_{L^{2}\left(\mathbb{T}^{2}\right)} \\
& \leq C\left\|f^{\varepsilon}-f-\varepsilon^{2} u\right\|_{L^{2}\left(\mathbb{T}^{2} \times \mathbb{R}^{2}\right)}\left\|f^{\varepsilon}-f\right\|_{L^{2}\left(\mathbb{T}^{2} \times \mathbb{R}^{2}\right)}
\end{aligned}
$$

Using the trivial inequality $\left\|f^{\varepsilon}-f\right\|_{L^{2}\left(\mathbb{T}^{2} \times \mathbb{R}^{2}\right)}^{2} \leq C\left(\left\|f^{\varepsilon}-f-\varepsilon^{2} u\right\|_{L^{2}\left(\mathbb{T}^{2} \times \mathbb{R}^{2}\right)}^{2}+\varepsilon^{4}\right)$ the formula (57) implies that

$$
\begin{array}{rl}
\left\|\left(f^{\varepsilon}-f-\varepsilon^{2} u\right)(t)\right\|_{L^{2}\left(\mathbb{T}^{2} \times \mathbb{R}^{2}\right)}^{2} & C C\left(\left\|f_{0}^{\varepsilon}-f_{0}\right\|_{L^{2}\left(\mathbb{T}^{2} \times \mathbb{R}^{2}\right)}^{2}+\varepsilon^{4}\right) \\
& +C \int_{0}^{t}\left\|\left(f^{\varepsilon}-f-\varepsilon^{2} u\right)(s)\right\|_{L^{2}\left(\mathbb{T}^{2} \times \mathbb{R}^{2}\right)}^{2} d s
\end{array}
$$

and thus by Gronwall lemma we deduce that

$$
\left\|\left(f^{\varepsilon}-f-\varepsilon^{2} u\right)(t)\right\|_{L^{2}\left(\mathbb{T}^{2} \times \mathbb{R}^{2}\right)}^{2} \leq C\left(\left\|f_{0}^{\varepsilon}-f_{0}\right\|_{L^{2}\left(\mathbb{T}^{2} \times \mathbb{R}^{2}\right)}^{2}+\varepsilon^{4}\right) .
$$

Finally we obtain for any $t \in[0, T]$ and $\varepsilon>0$

$$
\left\|\left(f^{\varepsilon}-f\right)(t)\right\|_{L^{2}\left(\mathbb{T}^{2} \times \mathbb{R}^{2}\right)}+\left\|\left(E^{\varepsilon}-E\right)(t)\right\|_{L^{2}\left(\mathbb{T}^{2}\right)} \leq C\left(\left\|f_{0}^{\varepsilon}-f_{0}\right\|_{L^{2}\left(\mathbb{T}^{2} \times \mathbb{R}^{2}\right)}+\varepsilon^{2}\right) .
$$

In particular taking $\varepsilon=\varepsilon_{k}$ one gets the convergences

$$
\lim _{k \rightarrow+\infty} f^{\varepsilon_{k}}=f \text { in } L^{\infty}(] 0, T\left[; L^{2}\left(\mathbb{T}^{2} \times \mathbb{R}^{2}\right)\right), \quad \lim _{k \rightarrow+\infty} E^{\varepsilon_{k}}=E \text { in } L^{\infty}(] 0, T\left[; L^{2}\left(\mathbb{T}^{2}\right)\right) .
$$




\section{Higher order approximation}

In the previous section we have justified rigorously the approximations

$$
f^{\varepsilon}=f+\mathcal{O}\left(\varepsilon^{2}\right) \text { in } L^{\infty}(] 0, T\left[; L^{2}\left(\mathbb{T}^{2} \times \mathbb{R}^{2}\right)\right), \quad E^{\varepsilon}=E+\mathcal{O}\left(\varepsilon^{2}\right) \text { in } L^{\infty}(] 0, T\left[; L^{2}\left(\mathbb{T}^{2}\right)\right)
$$

for initial conditions satisfying $f_{0}^{\varepsilon}=f_{0}+\mathcal{O}\left(\varepsilon^{2}\right)$ in $L^{2}\left(\mathbb{T}^{2} \times \mathbb{R}^{2}\right)$. We intend now to establish higher order approximations. More precisely we want to determine the correction terms $f_{1}, E_{1}$ such that

$$
f^{\varepsilon}=f+\varepsilon^{2} f_{1}+\mathcal{O}\left(\varepsilon^{4}\right), \quad E^{\varepsilon}=E+\varepsilon^{2} E_{1}+\mathcal{O}\left(\varepsilon^{4}\right) .
$$

Naturally, a complete theoretical study can be carried out following the same steps as before. However the purpose of the present section is only to provide the mathematical model governing $\left(f_{1}, E_{1}\right)$, based on formal considerations. In the sequel we denote by $\mathcal{T}$ the operator $p \cdot \nabla_{x}-B_{0,3}{ }^{\perp} p \cdot \nabla_{p}$ in the $L^{2}\left(\mathbb{T}^{2} \times \mathbb{R}^{2}\right)$ setting. By Proposition 3.4 for any $t \in[0, T]$ there are $\tilde{f}_{1}(t), \tilde{\tilde{f}}_{1}(t) \in L^{2}\left(\mathbb{T}^{2} \times \mathbb{R}^{2}\right)$ such that

$$
f_{1}(t)=\tilde{f}_{1}(t)+\tilde{\tilde{f}}_{1}(t), \quad \mathcal{T} \tilde{f}_{1}(t)=0,\left\langle\tilde{\tilde{f}}_{1}(t)\right\rangle=0, \quad \int_{\mathbb{T}^{2}} \int_{\mathbb{R}^{2}}\left(\tilde{f}_{1} \tilde{\tilde{f}}_{1}\right)(t, x, p) d p d x=0 .
$$

Therefore, by equation (30) we deduce that $\tilde{\tilde{f}}_{1}$ satisfies

$$
\mathcal{T} \tilde{\tilde{f}}_{1}(t)=-\partial_{t} f+E(t, x) \cdot \nabla_{p} f,\left\langle\tilde{\tilde{f}}_{1}(t)\right\rangle=0 .
$$

Recall that $\left\langle-\partial_{t} f+E(t, x) \cdot \nabla_{p} f\right\rangle=0$ and therefore, by Proposition 3.4 the function $\tilde{\tilde{f}}_{1}$ is well defined. It remains to determine the function $\tilde{f}_{1} \in \operatorname{Ker} \mathcal{T}$. Applying the gyro-average operator to (31) yields

$$
\partial_{t}\left\langle\tilde{f}_{1}\right\rangle-\left\langle E \cdot \nabla_{p} \tilde{f}_{1}\right\rangle-\left\langle E \cdot \nabla_{p} \tilde{\tilde{f}}_{1}\right\rangle-\left\langle E_{1} \cdot \nabla_{p} f\right\rangle=0
$$

By Proposition 3.2 we find the following expressions for the gyro-averages in the above equality

$$
\begin{gathered}
\left\langle\tilde{f}_{1}(t)\right\rangle(y, p)=\tilde{f}_{1}\left(t, y+\frac{{ }^{\perp} p}{B_{0,3}}, p\right) \text { since } \mathcal{T} \tilde{f}_{1}=0 \\
\left\langle E \cdot \nabla_{p} \tilde{f}_{1}\right\rangle(y, p)=-\left\langle\frac{{ }^{\perp} E(t)}{B_{0,3}}\right\rangle(y, p) \cdot \nabla_{x} \tilde{f}_{1}\left(t, y+\frac{{ }^{\perp} p}{B_{0,3}}, p\right) \text { since } \mathcal{T} \tilde{f}_{1}=0, \operatorname{div}_{x}{ }^{\perp} E=0 \\
\left\langle E_{1} \cdot \nabla_{p} f\right\rangle(y, p)=-\left\langle\frac{{ }^{\perp} E_{1}(t)}{B_{0,3}}\right\rangle(y, p) \cdot \nabla_{x} f\left(t, y+\frac{{ }^{\perp} p}{B_{0,3}}, p\right) \text { since } \mathcal{T} f=0, \operatorname{div}_{x}{ }^{\perp} E_{1}=0 .
\end{gathered}
$$

Therefore we obtain the following equation for $\tilde{f}_{1} \in \operatorname{Ker} \mathcal{T}$

$$
\begin{aligned}
\left(\partial_{t}+\left\langle\frac{{ }^{\perp} E(t)}{B_{0,3}}\right\rangle(y, p) \cdot \nabla_{x}\right) \tilde{f}_{1}\left(t, y+\frac{{ }^{\perp} p}{B_{0,3}}, p\right) & +\left\langle\frac{{ }^{\perp} E_{1}(t)}{B_{0,3}}\right\rangle(y, p) \cdot \nabla_{x} f\left(t, y+\frac{{ }^{\perp} p}{B_{0,3}}, p\right) \\
& =\left\langle E(t) \cdot \nabla_{p} \tilde{\tilde{f}}_{1}\right\rangle(y, p) .
\end{aligned}
$$

$\mathrm{RR} \quad \mathrm{n}^{\circ} 0123456789$ 
Notice also that if $\mathcal{T} \tilde{f}_{1}(0)=0$, then $\mathcal{T} \tilde{f}_{1}(t)=0$ for any $t \in \mathbb{R}_{+}$. Indeed, the equation (59) becomes in variables $(t, x, p)$

$$
\begin{aligned}
\left(\partial_{t}+\mathcal{V}_{E}\left(t, x-\frac{{ }^{\perp} p}{B_{0,3}}, p\right) \cdot \nabla_{x}\right) \tilde{f}_{1}(t, x, p) & +\mathcal{V}_{E_{1}}\left(t, x-\frac{{ }^{\perp} p}{B_{0,3}}, p\right) \cdot \nabla_{x} f(t, x, p) \\
& =\left\langle E(t) \cdot \nabla_{p} \tilde{\tilde{f}}_{1}\right\rangle\left(x-\frac{{ }^{\perp} p}{B_{0,3}}, p\right)
\end{aligned}
$$

where $\mathcal{V}_{E}(t, y, p)=\left\langle\frac{{ }^{\perp} E(t)}{B_{0,3}}\right\rangle(y, p), \mathcal{V}_{E_{1}}(t, y, p)=\left\langle\frac{{ }^{\perp} E_{1}(t)}{B_{0,3}}\right\rangle(y, p)$. By the definition of the gyro-average, the function $(y, p) \rightarrow\left\langle E(t) \cdot \nabla_{p} \tilde{\tilde{f}}_{1}\right\rangle(y, p)$ has radial symmetry with respect to $p \in \mathbb{R}^{2}$. Therefore by Proposition 3.1 the function $(x, p) \rightarrow\left\langle E(t) \cdot \nabla_{p} \tilde{\tilde{f}}_{1}\right\rangle\left(x-\frac{\perp_{p}}{B_{0,3}}, p\right)$ belongs to the kernel of $\mathcal{T}$. Similarly $\mathcal{V}_{E}\left(t, x-\frac{\perp p}{B_{0,3}}, p\right), \mathcal{V}_{E_{1}}\left(t, x-\frac{\perp p}{B_{0,3}}, p\right)$ belong to the kernel of $\mathcal{T}$ and thus

$$
\begin{gathered}
\mathcal{T}\left(\mathcal{V}_{E}\left(t, x-\frac{{ }^{\perp} p}{B_{0,3}}, p\right) \cdot \nabla_{x} \tilde{f}_{1}\right)=\mathcal{V}_{E}\left(t, x-\frac{{ }^{\perp} p}{B_{0,3}}, p\right) \cdot \nabla_{x} \mathcal{T} \tilde{f}_{1} \\
\mathcal{T}\left(\mathcal{V}_{E_{1}}\left(t, x-\frac{{ }^{\perp} p}{B_{0,3}}, p\right) \cdot \nabla_{x} f\right)=\mathcal{V}_{E_{1}}\left(t, x-\frac{{ }^{\perp} p}{B_{0,3}}, p\right) \cdot \nabla_{x} \mathcal{T} f=0 \text { since } \mathcal{T} f=0 .
\end{gathered}
$$

Applying now the operator $\mathcal{T}$ in (60) one gets easily

$$
\partial_{t} \mathcal{T} \tilde{f}_{1}+\mathcal{V}_{E} \cdot \nabla_{x} \mathcal{T} \tilde{f}_{1}=0
$$

saying that $\mathcal{T} \tilde{f}_{1}(t)=0$ for any $t \in \mathbb{R}_{+}$if $\mathcal{T} \tilde{f}_{1}(0)=0$. Finally we obtain that $\left(f_{1}=\tilde{f}_{1}+\tilde{\tilde{f}}_{1}, E_{1}\right)$ solve the system

$$
\left\{\begin{array}{l}
\mathcal{T} \tilde{\tilde{f}}_{1}(t)=-\partial_{t} f+E(t, x) \cdot \nabla_{p} f,\left\langle\tilde{\tilde{f}}_{1}(t)\right\rangle=0 \\
\partial_{t} \tilde{f}_{1}+\left\langle\frac{{ }^{\perp} E(t)}{B_{0,3}}\right\rangle\left(x-\frac{\perp p}{B_{0,3}}, p\right) \cdot \nabla_{x} \tilde{f}_{1}+\left\langle\frac{{ }^{E_{1}(t)}}{B_{0,3}}\right\rangle\left(x-\frac{\perp_{p}}{B_{0,3}}, p\right) \cdot \nabla_{x} f=\left\langle E \cdot \nabla_{p} \tilde{\tilde{f}}_{1}\right\rangle\left(x-\frac{{ }^{\perp}}{B_{0,3}}, p\right) \\
\mathcal{T} \tilde{f}_{1}(0)=0 \\
\operatorname{div}_{x} E_{1}=-\int_{\mathbb{R}^{2}}\left(\tilde{f}_{1}+\tilde{\tilde{f}}_{1}\right) d p, \operatorname{div}_{x}{ }^{\perp} E_{1}=0 .
\end{array}\right.
$$

Acknowledgement: The author is thankful to Prof. E. Sonnendrücker for fruitful discussions.

\section{References}

[1] A. Arseneev, Global existence of a weak solution of the Vlasov system of equations, U.R.S.S. Comp. Math. Phys. 15(1975) 131-143. 
[2] C. Bardos, P. Degond, Global existence for the Vlasov-Poisson equation in three space variables with small initial data, Ann. Inst. H. Poincaré, Anal. non linéaire 2(1985) 101-118.

[3] J. Batt, G. Rein, Global classical solutions of the periodic Vlasov-Poisson system in three dimensions, C. R. Acad. Sci. Paris, Sér. I 313(1991) 411-416.

[4] M. Bostan, Weak solutions for the Vlasov-Poisson initial-boundary value problem with bounded electric field, Chinese Ann. Math. 28(2007).

[5] M. Bostan, Mild solutions for the relativistic Vlasov-Maxwell system for laser-plasma interaction, Quart. Appl. Math. LXV(2007) 163-187.

[6] M. Bostan, T. Goudon, High-electric-field limit for the Vlasov-Maxwell-Fokker-Planck system, to appear in Ann. Inst. H. Poincaré, Anal. non linéaire.

[7] M. Bostan, The Vlasov-Maxwell system with strong initial magnetic field. Guidingcenter approximation, to appear in SIAM J. Multiscale Model. Simul.

[8] Y. Brenier, Convergence of the Vlasov-Poisson system to the incompressible Euler equations, Comm. Partial Differential Equations 25(2000) 737-754.

[9] Y. Brenier, N. Mauser, M. Puel, Incompressible Euler and e-MHD as scaling limits of the Vlasov-Maxwell system, Commun. Math. Sci. 1(2003) 437-447.

[10] H. Brezis, Analyse fonctionnelle, Masson 1983.

[11] F. Bouchut, F. Golse, C. Pallard, Classical solutions and the Glassey-Strauss theorem for the 3D Vlasov-Maxwell system, Arch. Ration. Mech. Anal. 170(2003) 1-15.

[12] J.A. Carrillo, S. Labrunie, Global solutions for the one-dimensional Vlasov-Maxwell system for laser-plasma interaction, Math. Models Methods Appl. Sci. 16(2006) 19-57.

[13] R. J. DiPerna, P.-L. Lions, Global weak solutions of the Vlasov-Maxwell system, Comm. Pure Appl. Math. XVII(1989) 729-757.

[14] E. Frénod, P.-A. Raviart, E. Sonnendrücker, Two-scale expansion of a singularly perturbed convection equation, J. Math. Pures Appl. 80(2001) 815-843.

[15] E. Frénod, E. Sonnendrücker, Homogenization of the Vlasov equation and of the Vlasov-Poisson system with strong external magnetic field, Asymptotic Anal. 18(1998) 193-213.

[16] E. Frénod, E. Sonnendrücker, Long time behavior of the Vlasov equation with a strong external magnetic field, Math. Models Methods Appl. Sci. 10(2000) 539-553.

[17] R. Glassey, J. Schaeffer, On the one and one-half dimensional relativistic VlasovMaxwell system, Math. Methods Appl. Sci. 13(1990) 169-179.

$\mathrm{RR} \quad \mathrm{n}^{\circ} 0123456789$ 
[18] R. Glassey, J. Schaeffer, The two and one-half dimensional relativistic Vlasov-Maxwell system, Comm. Math. Phy. 185(1997) 257-284.

[19] R. Glassey, J. Schaeffer, The relativistic Vlasov-Maxwell system in two space dimensions, Part I and II, Arch. Ration. Mech. Anal. 141(1998) 331-354 and 355-374.

[20] R. Glassey, W. Strauss, Singularity formation in a collisionless plasma could only occur at high velocities, Arch. Ration. Mech. Anal. 92(1986) 56-90.

[21] F. Golse, L. Saint-Raymond, The Vlasov-Poisson system with strong magnetic field, J. Math. Pures Appl. 78(1999) 791-817.

[22] F. Golse, L. Saint-Raymond, The Vlasov-Poisson system with strong magnetic field in quasineutral regime, Math. Models Methods Appl. Sci. 13(2003) 661-714.

[23] Y. Guo, The Vlasov-Maxwell-Boltzmann system near Maxwellians, Invent. Math. 153(2003) 593-630.

[24] E. Horst, On the classical solutions of the initial value problem for the unmodified nonlinear Vlasov equation, Math. Meth. Appl. Sci. 3(1981) 229-248.

[25] E. Horst, R. Hunze, Weak solutions of the initial value problem for the unmodified nonlinear Vlasov equation, Math. Meth. Appl. Sci. 6(1984) 262-279.

[26] S. Klainerman, G. Staffilani, A new approach to study the Vlasov-Maxwell system, Commun. Pure Appl. Anal. 1(2002) 103-125.

[27] P.-L. Lions, B. Perthame, Propagation of moments and regularity for the 3-dimensional Vlasov-Poisson system, Invent. Math. 105(1991) 415-430.

[28] K. Pfaffelmoser, Global classical solutions of the Vlasov-Poisson system in three dimensions for general initial data, J. Differential Equations 95(1992) 281-303.

[29] J. Schaeffer, Global existence of smooth solutions to the Vlasov-Poisson system in three dimensions, Comm. Partial Differential Equations 16(1991) 1313-1335.

[30] T. Ukai, S. Okabe, On the classical solution in the large time of the two dimensional Vlasov equations, Osaka J. Math. 15(1978) 245-261.

[31] H. Yu, Global classical solution of the Vlasov-Maxwell-Landau system near Maxwellians, J. Math. Phys. 45(2004) 4360-4376.

\section{Contents}

\begin{tabular}{lll}
\hline & Introduction & 3
\end{tabular}

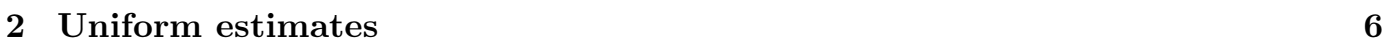


3 Formal derivation of the limit model 9

4 Well-posedness of the limit model 24

$\begin{array}{lll}5 & \text { Convergence results } & 28\end{array}$

6 Higher order approximation 31

$\mathrm{RR} \mathrm{n}^{\circ} 0123456789$ 


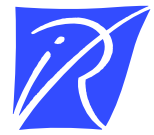

Unité de recherche INRIA Lorraine

LORIA, Technopôle de Nancy-Brabois - Campus scientifique 615, rue du Jardin Botanique - BP 101 - 54602 Villers-lès-Nancy Cedex (France)

Unité de recherche INRIA Futurs : Parc Club Orsay Université - ZAC des Vignes

4, rue Jacques Monod - 91893 ORSAY Cedex (France)

Unité de recherche INRIA Rennes : IRISA, Campus universitaire de Beaulieu - 35042 Rennes Cedex (France)

Unité de recherche INRIA Rhône-Alpes : 655, avenue de l'Europe - 38334 Montbonnot Saint-Ismier (France) Unité de recherche INRIA Rocquencourt : Domaine de Voluceau - Rocquencourt - BP 105 - 78153 Le Chesnay Cedex (France) Unité de recherche INRIA Sophia Antipolis : 2004, route des Lucioles - BP 93 - 06902 Sophia Antipolis Cedex (France) 